\title{
QUASILINEAR ELLIPTIC SYSTEMS
}

by

\author{
ALAN VAN LAIR, B.A., M.S .
}

A DISSERTATION

IN

\section{MATHEMATICS}

Submitted to the Graduate Faculty

of Texas Tech University in

Partial Fulfillment of

the Requirements for

the Degree of

DOCTOR OF PHILOSOPHY

Approved

Accepted

May, 1976 


$$
\begin{aligned}
& \text { AC } \\
& 80 ! \\
& 7=196 \\
& \text { No. } 18 \\
& \text { cup. } 2
\end{aligned}
$$

\section{ACKNOWLEDGMENTS}

I wish to thank Professor Wayne T. Ford, under whose direction this dissertation was written, for his advice and encouragement. Also, I wish to express my appreciation to the other members of my committee, Professors Vadim Komkov, Stanley R. Liberty, Monty J. Strauss, and John T. White for their helpful comments. 
TABLE OF CONTENTS

ACKNOWLEDGMENTS . . . . . . . . . . . . . . . ii

I. INTRODUCTION • . . . . . . . . . . . . . . . . 1

II. AUXILIARY RESULTS • . • • • • • • • • • • . 7

III. EXISTENCE • • • • . • • • • • • • . . . 11

IV. UNIQUENESS AND STABILITY • . • . • • • • • . 42

V. BOUNDEDNESS OF WEAK SOLUTIONS • • • • • • • 51

VI • BOUNDEDNESS OF THE HÖLDER NORM • • • • • • • • 67

VII. CONCLUSION • • • • • • • • • • • • • • • 71

LIST OF REFERENCES • • • • • • • • • • • • • • 72 
We shall study a boundary value problem for the second order differential system

$$
\text { (1.1) } \begin{aligned}
\sum_{\ell=1}^{n} \frac{\partial}{\partial x_{\ell}}\{ & \left.\sum_{j=1}^{N} \sum_{m=1}^{n} C_{i j}^{\ell m}[x, U(x)] \frac{\partial U^{j}}{\partial x_{m}}(x)+B_{i}^{\ell}[x, U(x)]\right\} \\
& +F_{i}[x, U(x)]=0,1 \leq i \leq N
\end{aligned}
$$

where $x=\left(x_{1}, x_{2}, \ldots, x_{n}\right)$ lies in a bounded open connected set $\Omega$ in $E_{n}$, and a solution $U=\left(U^{l}, U^{2}, \ldots, U^{N}\right)$ maps $\Omega$ into $E_{N}$ subject to the boundary conditions

$$
U^{i}(x)=0, x \varepsilon S \equiv \text { boundary of } \Omega
$$

Formulations will be abbreviated when convenient through the combined use of summation convention on repeated indices, $\mathrm{U}, \mathrm{m}$ for $\partial \mathrm{U} / \partial \mathrm{x}_{\mathrm{m}}$, and suppression of arguments. Thus, the differential system (1.1) becomes

$$
\left(c_{i j}^{l m} U_{, m}^{j}+B_{i}^{l}\right), l+F_{i}=0
$$

The Euclidean norm of appropriate dimension will be 
denoted by $|\cdot|$. Thus, $|x|=\sum_{m=1}^{n}\left(x_{m}\right)^{2},|U|^{2}=\sum_{i=1}^{N}\left(U^{i}\right)^{2}$, $|B|^{2}=\sum_{\ell=1}^{n} \sum_{i=1}^{N}\left(B_{i}^{\ell}\right)^{2},\left|U_{x}\right|^{2}=\sum_{m=1}^{n} \sum_{i=1}^{N}\left(U_{, m}^{i}\right)^{2}$, and $|c|^{2}=\sum_{\ell=1}^{n} \sum_{m=1}^{n} \sum_{i=1}^{N} \sum_{j=1}^{N}\left(c_{i j}^{\ell m}\right)^{2}$. Summation convention allows these norms to be respectively written as $|x|^{2}=\left(x_{m}\right)^{2}$, $|U|^{2}=\left(U^{i}\right)^{2},|B|^{2}=\left(B_{i}^{l}\right)^{2},\left|U_{x}\right|^{2}=\left(U^{i}, m^{2}\right.$, and $|c|^{2}=\left(c_{i j}^{l m}\right)^{2}$

If $p \geq 1$, let $L_{p}(\Omega)$ be the Banach space of measurable functions from $\Omega$ to $E_{N}$ with finite norm

$$
\left.|| \mathrm{U}\right|_{\mathrm{p}, \Omega} ^{\mathrm{p}}=\int_{\Omega}|\mathrm{u}(\mathrm{x})|^{\mathrm{p}} \mathrm{dx}
$$

If $\mathrm{p}=2,(\cdot, \cdot)_{2, \Omega}$ will denote the natural inner product induced by (1.4), and we let $\mathrm{H}^{1}(\Omega)$ denote the Hilbert space induced by

$$
\begin{aligned}
(\mathrm{U}, \mathrm{V})_{0, \Omega} & =(\mathrm{U}, \mathrm{V})_{2, \Omega}+\left(\mathrm{U}, \mathrm{m}^{,} \mathrm{V}, \mathrm{m}\right)^{\prime}, \Omega \\
& \equiv \int_{\Omega} \mathrm{U}^{\mathrm{i}} \mathrm{v}^{\mathrm{i}} \mathrm{dx}+\int_{\Omega} \mathrm{U}_{, \mathrm{m}}^{\mathrm{i}} \mathrm{v}^{\mathrm{i}} \mathrm{m}
\end{aligned}
$$

Also, let $\mathrm{H}_{0}^{l}(\Omega)$ be the completion of $\mathrm{C}_{0}^{\infty}(\Omega)$ in the $\mathrm{H}^{1}(\Omega)$ norm.

We shall say that the function $U$ from $\Omega$ into $E_{N}$ satisfies 
a Hölder condition with exponent $\alpha$, where $\alpha \varepsilon(0,1]$, and with Hölder constant $M$ in the region $\bar{\Omega}$ (closure of $\Omega$ ) if

$$
\left|\mathrm{U}^{\mathrm{i}}\right|_{(\alpha), \Omega} \equiv \sup \rho^{-\alpha} \operatorname{osc}\left\{\mathrm{U}^{\mathrm{i}}, \mathrm{K}_{\rho} \cap \Omega\right\} \equiv \mathrm{M}<\infty
$$

for each $i=1, \ldots, N$ where $k_{\rho}$ denotes an arbitrary open sphere of radius $\rho$ in $E_{n}$ and $\operatorname{osc}\left\{U^{i}, K_{\rho} \cap \Omega\right\}$ is the difference between ess $\sup \left\{U^{i}(x): x \in K_{\rho} \cap \Omega\right\}$ and ess $\inf \left\{U^{i}(x):\right.$ $\left.x \in K_{\rho} \cap \Omega\right\}$. The supremum in $(1.6)$ is taken over $0<\rho \leq \rho_{0}$. $\mathrm{C}_{0, \alpha}(\bar{\Omega})$ is the Banach space of elements consisting of all bounded functions $U$ that are continuous in $\Omega$ such that $|U|_{(\alpha), \Omega}^{2} \equiv \sum_{i=1}^{N}\left(\left|U^{i}\right|(\alpha), \Omega\right)^{2}$ is finite. The norm in $C_{0, \alpha}(\bar{\Omega})$ is defined as

$$
|\mathrm{U}|_{\alpha, \Omega} \equiv \sup _{\mathrm{x} \varepsilon \Omega}|\mathrm{U}(\mathrm{x})|+|\mathrm{U}|(\alpha), \Omega
$$

The domain of definition of the elements $C_{0, \alpha}(\bar{\Omega})$ can be thought of as extended to $S$ in such a way as to be continuous from within.

A function belongs to $c_{0, \alpha}(\Omega)$ if it belongs to $c_{0, \alpha}(\bar{\Omega})$ for all $\overline{\Omega^{\top}} \subset \Omega$.

We denote by $\mathrm{B}(\Omega, \mathrm{M}, \gamma, \delta, 1 / q)$ the class of real-valued functions $V$ in $H^{1}(\Omega)$ with ess $\sup _{x \in \Omega}|V(x)| \leq M$ such that, for both $\mathrm{V}$ and $-\mathrm{V}$, the following inequalities are valid in an arbitrary open sphere $\mathrm{k}_{\rho} \cap \Omega$ for arbitrary $\sigma \in(0,1)$ : 


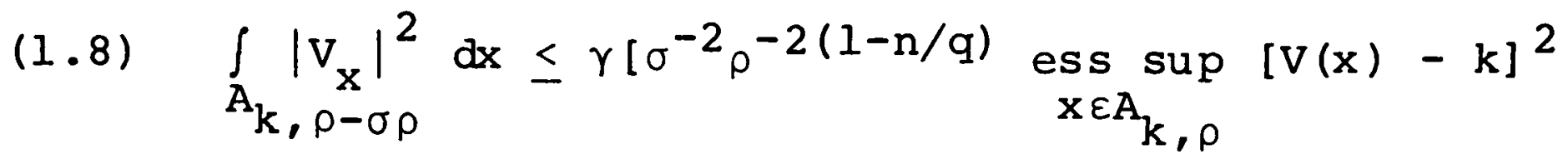

$$
+1]\left(\text { mes } A_{k, \rho}\right)^{1-2 / q}
$$

for $\mathrm{k} \geq \operatorname{ess} \sup _{\mathrm{x} \in \mathrm{K}} \mathrm{V}(\mathrm{x})-\delta$ where $A_{\mathrm{k}, \rho} \equiv\left\{\mathrm{x} \varepsilon \mathrm{K}_{\rho} \mid \mathrm{V}(\mathrm{x})>\mathrm{k}\right\}$, $K_{\rho-\sigma \rho}$ is a sphere concentric with $K_{\rho}$, and mes $G \equiv$ measure of G. We may assume the radii of the spheres $\rho$ in (1.8) do not exceed some positive number $\bar{\rho}$. The parameters $M, \gamma$, and $\delta$ are arbitrary positive numbers and $2 \leq \mathrm{n}<\mathrm{q}<\infty$.

For applications, it will be useful to note the following: If, in an open sphere $K_{\rho}$, a function $V$ satisfies the inequalities

$$
\begin{aligned}
\int_{A_{k}, \rho}\left|v_{x}\right|^{2} \zeta^{2} d x \leq \gamma^{\prime}\left[\int_{A_{k}, \rho}(V-k)^{2}\left|\zeta_{x}\right|^{2} d x\right. \\
\left.+\left(\operatorname{mes} A_{k, \rho}\right)^{1-2 / q}\right]
\end{aligned}
$$

for $k \geq \operatorname{ess} \sup _{x \in K_{\rho}} V(x)-\delta$ and for an arbitrary nonnegative smooth function $\zeta$ that vanishes on the surface of $\mathrm{K}_{\rho}$, then inequality (1.8) is valid for $k_{\rho}$ and an arbitrary concentric sphere $k_{\rho-\sigma \rho}$ where $\sigma \in(0,1)$. (See $\left.[8, \mathrm{p} .82].\right)$

We denote by $B(\bar{\Omega}, M, \gamma, \delta, 1 / q)$ the class of real-valued functions $V$ in $B(\Omega, M, \gamma, \delta, l / q)$ that, together with their negatives, satisfy inequalities (1.8) for the spheres $k_{\rho}$ 


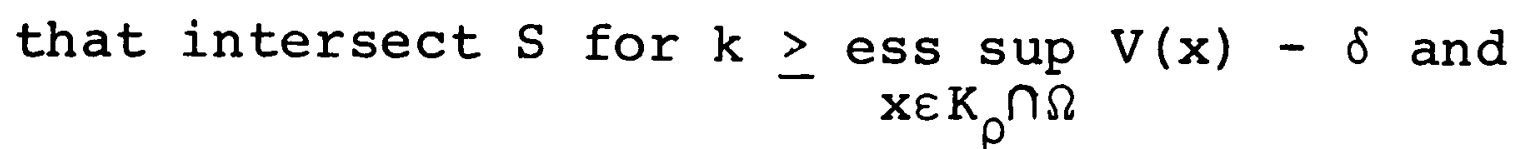

$k \geq \underset{x \varepsilon K_{\rho} \cap S}{\operatorname{ess} \sup } V(x)$. The regions of intersection $A_{k, \rho}$ for the spheres intersecting $S$ in (1.8) are defined as the sets of points in $k_{\rho} \cap \Omega$ at which $V(x)>k$.

Finally, we shall say that the boundary $S$ of a region $\Omega$ satisfies condition (A) if there exist two positive numbers $a_{o}$ and $\theta_{0}$ such that, for an arbitrary sphere $k_{\rho}$ with center on $\mathrm{S}$ of radius $\rho \leq \mathrm{a}_{0}$ and for an arbitrary component $\hat{\Omega}_{\rho}$ of the intersection of the sphere $\mathrm{K}_{\rho}$ with $\Omega$, the inequality

$$
\text { mes } \hat{\Omega}_{\rho} \leq\left(1-\theta_{0}\right) \text { mes } K_{\rho}
$$

holds. We shall use condition (A) in Chapter VI only. For the other chapters, we simply assume that $S$ is sufficiently smooth to ensure that integration by parts is valid.

Solution of (1.1) will be developed and considered in a weak sense. If $\mathrm{z} \varepsilon \mathrm{H}_{0}^{l}(\Omega)$, multiply (1.1) by $\mathrm{z}^{i}$ and obtain (sum by convention)

$$
\int_{\Omega} z^{i}\left(C_{i j}^{\ell m} U_{, m}^{j}+B_{i}^{\ell}\right), \ell d x+\int_{\Omega} z^{i} F_{i} d x=0 .
$$

This implies

$$
\int_{\Omega} z^{i}{ }_{l} C_{i j}^{\ell m} U_{, m}^{j} d x+\int_{\Omega} z_{,}^{i},^{B_{i}^{\ell}} d x-\int_{\Omega} z^{i} F_{i} d x=0
$$

as a motivation for a definition of weak solutions of (1.1) and (1.2). 
DEFINITION 1.1. We shall say that $U \in H_{0}^{l}(\Omega)$ is a weak solution of (1.1) and (1.2) if (1.12) is valid whenever $\mathrm{Z} \varepsilon \mathrm{H}_{0}^{1}(\Omega)$.

The main result of this paper is a proof of the existence of a weak solution to (1.1) and (1.2) with minimal conditions on the auxiliary functions C, B, and F. Visik [13] studied existence of weak solutions as well as uniqueness for higher order $(\geq 2)$ quasilinear strongly elliptic systems but required considerable smoothness conditions on the auxiliary functions. Browder $[2,3]$ also considered existence of weak solutions and uniqueness for similar systems but imposed monotonicity constraints on the auxiliary functions with some growth rate restrictions.

We shall also consider uniqueness of our weak solution, but stronger restrictions on the auxiliary functions are necessarily needed as will be shown by an example. In addition, stability with respect to variations in $C, B$, and F will be studied. We shall conclude with a proof of the boundedness of the Hölder norms of the weak solutions under more restrictive conditions on $C, B$, and $F$ than those needed for existence. This regularity argument uses essentially the same method as that of Ladyzhenskaya and Ural'tseva [8, Chapter 4] for a single equation. 
CHAPTER II

AUXILIARY RESULTS

Certain known results and easily proved propositions that will be needed throughout the paper are collected in this chapter.

DEFINITION 2.1. Let $\mathrm{Y}$ be a Banach space. A set $\mathrm{D} \subset \mathrm{Y}$ is relatively compact in $\mathrm{Y}$ if $\overline{\mathrm{D}}$ is compact in $\mathrm{Y}$.

DEFINITION 2.2. Let $\mathrm{X}$ and $\mathrm{Y}$ be Banach spaces. A mapping $f: X \rightarrow Y$ is compact if it is continuous and maps bounded sets into relatively compact sets, i.e., if $\mathrm{B} \subset \mathrm{X}$ is bounded, then $f(B)$ is relatively compact in $Y$.

THEOREM 2.1. (Rellich) Every bounded set in $\mathrm{H}_{0}^{1}(\Omega)$ is relatively compact in $\mathrm{L}_{2}(\Omega)$.

PROOF. (See $[1$, p. $194 ; 9]$.

THEOREM 2.2. (Schauder) Let A be a closed convex bounded set contained in the Banach space $X$. Every compact mapping $\mathrm{f}: \mathrm{A} \rightarrow \mathrm{A}$ has a fixed point.

PROOF. (See $[12$, p. 96].)

THEOREM 2.3. Let $\mathrm{V}$ be an arbitrary real-valued function in the class $B(\Omega, M, \gamma, \delta, l / q)$, and let $k_{\rho_{0}}$ be a sphere of radius $\rho_{0} \leq 1$ belonging to $\Omega$. Then, for an arbitrary sphere 
$K_{\rho}$, where $\rho \leq \rho_{0}$ that is concentric with $K_{\rho_{0}}$, the oscillation of $\mathrm{V}$ in $\mathrm{K}_{\rho}$ satisfies the inequality

$$
\operatorname{osc}\left\{\mathrm{V}, \mathrm{K}_{\rho}\right\} \leq \mathrm{c}\left(\rho / \rho_{0}\right)^{\alpha}
$$

where

$$
\begin{aligned}
& \alpha=\min \left\{-\log _{4}\left(1-2^{1-s}\right), 1-n / q\right\}, \\
& c=4^{\alpha} \max \left\{2 M, 2^{s} \rho_{0}^{1-n / q}\right\}
\end{aligned}
$$

and the number $\mathrm{s} \geq \log _{2}(2 \mathrm{M} / \delta)+3$.

PROOF. (See $[8$, p. 90].)

THEOREM 2.4. If $S$ satisfies condition (A) and if the function $V$ in $B(\bar{\Omega}, M, \gamma, \delta, l / q)$ satisfies on $S$ a Hölder condition, more precisely, if

$$
\operatorname{Osc}\left\{\mathrm{V}, \mathrm{S} \cap \mathrm{K}_{\rho}\right\} \leq \mathrm{L} \rho^{\varepsilon}, \varepsilon>0
$$

for spheres $K_{\rho}$ (where $\rho \leq a_{0}$ ) with centers on $S$, then $V$ satisfies a Hölder condition in $\bar{\Omega}$; that is, for arbitrary $\mathrm{K}_{\rho}$

$$
\operatorname{osc}\left\{\mathrm{V}, \mathrm{k}_{\rho} \cap \Omega\right\} \leq \mathrm{c} \rho^{\alpha} \text {. }
$$

Here, the constants $c$ and $\alpha$ are determined only by the parameters $M, \delta, \gamma$, and $l / q$ of the class $B(\bar{\Omega}, M, \gamma, \delta, l / q)$, by $L$ and $\varepsilon$ in (2.4), and by the constants $a_{0}$ and $\theta_{0}$ in condition (A) on S.

PROOF. (See $[8$, p. 92].) 
IEMMA 2.1. Let $\mathrm{U}$ be an arbitrary function in $\mathrm{H}_{0}^{1}(\Omega)$. Then

$$
\left.|| \mathrm{U}\right|_{\mathrm{p}} \leq \mathrm{M}_{\mathrm{O}}(\operatorname{mes} \Omega)^{1 / p-(q-2) / 2 \mathrm{q}}|| \mathrm{U}_{\mathrm{x}} \|_{2, \Omega}
$$

where $1 \leq \mathrm{p} \leq 2 \mathrm{q} /(\mathrm{q}-2)$ with $\mathrm{q} \geq \mathrm{n}$ if $\mathrm{n} \geq 3, \mathrm{q}>2$ if $\mathrm{n}=2$, and the constant $M_{0}$ is determined only by $p$ and $n$. PROOF. (See $[8$, p. 45].)

COROLLARY 2.1. The norms $\left.|| \mathrm{U}\right|_{0, \Omega}$ and $\left.|| \mathrm{U}_{\mathbf{x}}\right|_{2, \Omega}$ are equivalent on $\mathrm{H}_{0}^{1}(\Omega)$.

PROOF. From Lemma 2.1 with $\mathrm{p}=2$, we get the inequalities

$$
\text { (2.7) } \quad \mid \mathrm{U}_{\mathrm{x}}\left\|_{2, \Omega}^{2} \leq\right\| \mathrm{U}\left\|_{2, \Omega}^{2}+\right\| \mathrm{U}_{\mathrm{x}}\left\|_{2, \Omega}^{2} \leq\left(1+\mathrm{b}^{2}\right)\right\| \mathrm{U}_{\mathrm{x}} \|_{2, \Omega}^{2}
$$

where $\mathrm{b}^{2} \equiv \mathrm{M}_{\mathrm{O}}^{2}$ (mes $\left.\Omega\right)^{2 / \mathrm{n}}$ with $\mathrm{M}_{\mathrm{O}}$ from Lemma 2.1 and the proof is complete.

LEMMA 2.2. Suppose a and $c$ are nonnegative numbers and $\varepsilon$ is an arbitrary positive number. If $0<\lambda<1$, then

$$
a^{\lambda} c^{1-\lambda} \leq \varepsilon a+(1-\lambda)(\lambda / \varepsilon)^{\lambda /(1-\lambda)} c .
$$

PROOF. $\quad a^{\lambda} c^{1-\lambda}=(a \varepsilon / \lambda)^{\lambda}\left[(\lambda / \varepsilon)^{\lambda /(1-\lambda)} c\right]^{1-\lambda}$

$$
\leq \varepsilon a+(1-\lambda)(\lambda / \varepsilon)^{\lambda /(1-\lambda)} \mathrm{c}
$$

where the last inequality is from [11, p. 112], and the proof is complete.

Note that in the special case of $a=q^{2}, c=d^{2}$, and $\lambda=1 / 2,(2.8)$ reduces to 
$(2.9)$

$$
|q d| \leq \varepsilon q^{2}+(1 / 4 \varepsilon) d^{2}
$$

We conclude Chapter 2 with a theorem that is useful in showing boundedness of weak solutions.

THEOREM 2.5. Suppose that a real-valued function $\mathrm{V}$ in $H^{l}(\Omega)$ has bounded ess $\sup _{x \in \Omega} V(x)$ and that for $k \geq \hat{k} \geq$ ess sup $V(x)$ satisfies the inequalities $\mathrm{x} \varepsilon \mathrm{S}$

(2.10) $\quad \int_{A_{k}}\left|v_{x}\right| d x \leq \gamma\left[\int_{A_{k}}(V-k)^{2} d x+\sum_{i=1}^{N} k^{\alpha_{i}}\left(\operatorname{mes} A_{k}\right)^{s}\right]$ where $s=1-2 / n+\varepsilon_{i}$. Also, $\gamma, \alpha_{i}, \varepsilon_{i}$, and $N_{0}$ are positive constants and $A_{k} \equiv\{x \in \Omega \mid V(x)>k\}$. Suppose also that $2 \leq \alpha_{i}<2\left(1+\varepsilon_{i}\right)$. Then, ess $\sup _{x \in \Omega} V(x)$ does not exceed some constant that depends only on $\gamma_{,} N_{0^{\prime}} \alpha_{i}, \varepsilon_{i}$, $n, \hat{k}$, mes $\Omega$, and the norm $\|v\|_{1, A_{\hat{k}}}$ with $i=1, \ldots, N_{0}$. PROOF. (See $[8, \mathrm{p} .73]$. 
We now impose conditions on the auxiliary functions of (1.1) in order to obtain the existence of a weak solution of (1.1) and (1.2). After proving existence under the conditions of Assumption 3.1, we will relax some of these restrictions and prove existence under the more general conditions.

ASSUMPTION 3.1. There exists a positive constant $\lambda$ such that

(3.1) $\quad C_{i j}^{l m} y_{l}^{i} y_{m}^{j} \geq \lambda|y|^{2}, y \in E_{n N}$.

Moreover, $M_{C}$ is a positive constant such that

$$
\left|C_{i j}^{l m}[x, U]\right|^{2} \leq M_{C}^{2}, x \in \Omega, U \in E_{N}
$$

Also, there exist nonnegative constants $\varepsilon_{0^{\prime}} \varepsilon_{1}, M_{F}$, and $M_{B}$ such that

(3.3) $\varepsilon_{0}+\varepsilon_{1}<\lambda^{2}\left[1+b^{2}\right]^{-2}$,

(3.4) $|\mathrm{F}[\mathrm{x}, \mathrm{U}]|^{2} \leq \varepsilon_{\mathrm{O}}|\mathrm{U}|^{2}+\mathrm{M}_{\mathrm{F}}, \mathrm{x} \varepsilon \Omega, \mathrm{U} \varepsilon \mathrm{E}_{\mathrm{N}^{\prime}}$ (3.5) $|\mathrm{B}[\mathrm{x}, \mathrm{U}]|^{2} \leq \varepsilon_{1}|\mathrm{U}|^{2}+\mathrm{M}_{\mathrm{B}}, \mathrm{x} \varepsilon \Omega, \mathrm{U} \varepsilon \mathrm{E}_{\mathrm{N}}$. Here, $b$ is from (2.7). Finally, $F_{i}, B_{i}^{l}$, and $C_{i j}^{l m}$ are continuous maps from $\mathrm{L}_{2}(\Omega)$ into $\mathrm{L}_{2}(\Omega)$; i.e., if $h(V) \equiv F_{i}[\cdot, V(\cdot)]$, then we assume that the mapping $\mathrm{h}$ is continuous. 
We shall use the Schauder Fixed Point Theorem to prove the existence of a weak solution of (1.1) and (1.2). However, we first consider the problem of proving existence of a solution to a linear system.

Let $\mathrm{V}$ be a fixed element of $\mathrm{L}_{2}(\Omega)$. We wish to prove the existence of a unique $W \varepsilon H_{0}^{l}(\Omega)$ such that

(3.6) $\int_{\Omega} z^{i}, l^{C_{i j}^{\ell m}}[x, v] w_{,}^{j} d x+\int_{\Omega} z^{i}, l^{B_{i}^{\ell}}[x, v] d x=\int_{\Omega} z^{i} F_{i}[x, v] d x$ for every $\mathrm{Z} \varepsilon \mathrm{H}_{0}^{l}(\Omega)$. For this purpose we define the following functionals on $\mathrm{H}_{0}^{l}(\Omega) \times \mathrm{H}_{0}^{1}(\Omega)$ and $\mathrm{H}_{0}^{1}(\Omega)$, respectively.

$$
\begin{aligned}
& L[z, w] \equiv \int_{\Omega} z_{, l}^{i} C_{i j}^{\ell m}[x, V] w_{, m}^{j} d x . \\
& P[z] \equiv-\int_{\Omega} z_{, l}^{i} B_{i}^{\ell}[x, v] d x+\int_{\Omega} z^{i} F_{i}[x, V] d x .
\end{aligned}
$$

We now prove a series of propositions concerning the functionals $P$ and $L$.

PROPOSITION 3.1. $\mathrm{P}$ is a bounded linear functional on $\mathrm{H}_{0}^{l}(\Omega)$.

PROOF. Clearly, $P$ is a linear functional on $\mathrm{H}_{0}^{1}(\Omega)$, and we need only prove boundedness. From Schwarz's inequality, we get

$$
\text { (3.9) } \begin{aligned}
|\mathrm{P}[\mathrm{z}]| \leq & \int_{\Omega}\left|\mathrm{z}_{\mathrm{x}}\right||\mathrm{B}[\mathrm{x}, \mathrm{V}]| \mathrm{dx}+\int_{\Omega}|\mathrm{z}||\mathrm{F}[\mathrm{x}, \mathrm{V}]| \mathrm{dx} \\
\leq & || \mathrm{z}_{\mathrm{x}}||_{2, \Omega}|| \mathrm{B}[\cdot, \mathrm{V}(\cdot)]||_{2, \Omega} \\
& +|| \mathrm{z} \|\left._{2, \Omega}|| \mathrm{F}[\cdot, \mathrm{V}(\cdot)]\right|_{2, \Omega}
\end{aligned}
$$




$$
\begin{aligned}
& \leq\left(|| \mathrm{B}[\cdot, \mathrm{V}(\cdot)]\left\|_{2, \Omega}+\right\| \mathrm{F}[\cdot, \mathrm{V}(\cdot)] \|_{2, \Omega}\right) \\
& \times\left(\left.|| z\right|_{2, \Omega}+|| z_{x} \|_{2, \Omega}\right) \\
& \equiv M(B, F, V)\left\{|| z||_{2, \Omega}+|| z_{x} \|_{2, \Omega}\right\} \\
& \leq\left. 2 M(B, F, V)|| z\right|_{0, \Omega^{\prime}}
\end{aligned}
$$

and the proof is complete.

PROPOSITION 3.2. L is a bilinear functional on $H_{0}^{l}(\Omega) \times H_{0}^{l}(\Omega)$, and there exist positive constants $\lambda_{0}$ and $\lambda_{\infty}$ such that

and

$$
|\mathrm{L}[\mathrm{Z}, \mathrm{W}]| \leq\left.\lambda_{\infty}|| \mathrm{z}\right|_{0, \Omega}|| \mathrm{W}||_{0, \Omega}
$$

$$
\lambda_{0}|| z||_{0, \Omega}^{2} \leq L[z, z]
$$

for all $\mathrm{Z}$ and $\mathrm{W}$ in $\mathrm{H}_{0}^{\mathrm{l}}(\Omega)$.

PROOF. Clearly, $L$ is a bilinear functional on

$\mathrm{H}_{0}^{1}(\Omega) \times \mathrm{H}_{0}^{1}(\Omega)$, and we need only prove the existence of $\lambda_{0}$ and $\lambda_{\infty}$. From Schwarz's inequality, we have

$$
\begin{aligned}
|\mathrm{L}[\mathrm{z}, \mathrm{w}]| & \leq \int_{\Omega}\left|\mathrm{z}_{\mathrm{x}}\right||\mathrm{C}[\mathrm{x}, \mathrm{v}]|\left|\mathrm{w}_{\mathrm{x}}\right| \mathrm{dx} \\
& \leq \|\left|\mathrm{z}_{\mathrm{x}}\right||\mathrm{C}[\mathrm{x}, \mathrm{v}]|||_{2, \Omega}|| \mathrm{w}_{\mathrm{x}}||_{2, \Omega} \\
& \leq\left.\mathrm{M}_{\mathrm{C}}|| \mathrm{z}\right|_{0, \Omega}|| \mathrm{w}||_{0, \Omega} .
\end{aligned}
$$

Thus, let $\lambda_{\infty}=M_{C}$ where $M_{C}$ is from (3.2), and (3.10) is proved. For the proof of (3.11), we use (3.1) to get 


$$
\lambda\left\|z_{x}\right\|_{2, \Omega}^{2} \leq L[z, z]
$$

for all $\mathrm{Z}$ in $\mathrm{H}_{0}^{1}(\Omega)$. However, (2.7) combined with (3.13) implies

$$
\text { (3.14) } \quad \lambda\left(1+\mathrm{b}^{2}\right)^{-1}|| \mathrm{z}\left\|_{0, \Omega}^{2} \leq \lambda|| \mathrm{z}_{\mathrm{x}}\right\|_{2, \Omega}^{2} \leq \mathrm{L}[\mathrm{z}, \mathrm{z}]
$$

Thus, define $\lambda_{0}=\lambda\left(1+b^{2}\right)^{-1}$ where $b$ is from (2.7), and the proof is complete.

PROPOSITION 3.3. There exists a unique $W$ in $H_{0}^{1}(\Omega)$ such that

$$
\mathrm{L}[\mathrm{Z}, \mathrm{W}]=\mathrm{P}[\mathrm{Z}]
$$

for all $\mathrm{z}$ in $\mathrm{H}_{0}^{1}(\Omega)$. Thus, $\mathrm{W}$ is the unique solution of $(3.6)$.

PROOF. Uniqueness is clear from inequality (3.11). For the existence, we observe that Proposition 3.1 and F. Riesz' representation theorem [14, p. 90] imply the existence of a unique $\mathrm{f}$ in $\mathrm{H}_{0}^{l}(\Omega)$ such that

$$
P[Z]=(Z, f) 0, \Omega
$$

for all $\mathrm{z}$ in $\mathrm{H}_{0}^{l}(\Omega)$. Also, Proposition 3.2 and the LaxMilgram Theorem [14, p. 92] imply the existence of a unique determined bounded linear operator $\mathrm{T}: \mathrm{H}_{0}^{1}(\Omega)+\mathrm{H}_{0}^{l}(\Omega)$ such that

$$
\mathrm{L}[\mathrm{Z}, \mathrm{T}(\mathrm{U})]=(\mathrm{Z}, \mathrm{U})_{0, \Omega}
$$

for all $\mathrm{Z}$ and $\mathrm{U}$ in $\mathrm{H}_{0}^{\mathrm{I}}(\Omega)$. In particular, (3.17) must be true for $U=f$, and this means 


$$
L[Z, T(f)]=(Z, f)_{0, \Omega}=P[Z]
$$

for all $\mathrm{Z}$ in $\mathrm{H}_{0}^{\mathrm{l}}(\Omega)$. Thus, $\mathrm{W} \equiv \mathrm{T}(f)$ is the desired solution, and the proof is complete.

Now Proposition 3.3 enables us to use $(3.6)$ to define a mapping $\Phi: \mathrm{L}_{2}(\Omega) \rightarrow \mathrm{H}_{0}^{\mathrm{l}}(\Omega)$ by $\mathrm{W}=\Phi(\mathrm{V})$. In order to prove the existence of a solution $U$ to (1.1) and (1.2), we need only show that $\Phi$ has a fixed point. To accomplish this, we prove a series of lemmas concerning the mapping $\Phi$.

LEMMA 3.1. The mapping $\Phi: \mathrm{L}_{2}(\Omega) \rightarrow \mathrm{L}_{2}(\Omega)$ is continuous whenever Assumption 3.1 is valid.

PROOF. Suppose $\mathrm{V}_{\mathrm{k}} \rightarrow \mathrm{V}$ in $\mathrm{L}_{2}(\Omega)$ as $\mathrm{k} \rightarrow \infty$. It suffices to show $\left\|\left[\Phi\left(\mathrm{V}_{\mathrm{k}}\right)-\Phi(\mathrm{V})\right]_{\mathrm{x}}\right\|_{2, \Omega} \rightarrow 0$ as $\mathrm{k} \rightarrow \infty$ since then Lemma 2.1 implies $\Phi\left(\mathrm{V}_{\mathrm{k}}\right) \rightarrow \Phi(\mathrm{V})$ in $\mathrm{L}_{2}(\Omega)$. With $\mathrm{W}_{\mathrm{k}} \equiv \Phi\left(\mathrm{V}_{\mathrm{k}}\right)$, $W \equiv \Phi(V)$, and the use of the corresponding integral identities involving $w$ and $w_{k}$, we get

$$
\text { (3.19) } \begin{aligned}
& \int_{\Omega} z_{, l}^{i}\left[C_{i j}^{l m}[x, v] w_{, m}^{j}-C_{i j}^{l m}\left[x, v_{k}\right] w_{k, m}^{j}\right] d x \\
+ & \int_{\Omega} z_{, l}^{i}\left[B_{i}^{l}[x, v]-B_{i}^{l}\left[x, v_{k}\right]\right] d x \\
= & \int_{\Omega} z^{i}\left[F_{i}[x, v]-F_{i}\left[x, v_{k}\right]\right] d x
\end{aligned}
$$

for each $\mathrm{z}$ in $\mathrm{H}_{0}^{1}(\Omega)$. This implies

(3.20) $\int_{\Omega} z_{, l}^{i} c_{i j}^{l m}\left[x, v_{k}\right]\left[w_{, m}^{j}-w_{k, m}^{j}\right] d x$ 


$$
\begin{aligned}
& +\int_{\Omega} z_{, l}^{i}\left[C_{i j}^{l m}[x, v]-C_{i j}^{\ell m}\left[x, v_{k}\right]\right] w_{, m}^{j} d x \\
& +\int_{\Omega} z_{, l}^{i}\left[B_{i}^{l}[x, v]-B_{i}^{l}\left[x, v_{k}\right]\right] d x \\
& =\int_{\Omega} z^{i}\left[F_{i}[x, v]-F_{i}\left[x, v_{k}\right]\right] d x .
\end{aligned}
$$

In (3.20), we put $z=W-W_{k}$. Using Assumption 3.1, we get

$$
\begin{aligned}
& \text { (3.21) } \lambda \int_{\Omega}\left|z_{x}\right|^{2} d x \\
& \leq \int_{\Omega}\left|z_{x}\right|\left|C[x, v]-c\left[x, v_{k}\right]\right|\left|w_{x}\right| d x \\
& +\int_{\Omega}\left|z_{x}\right|\left|B[x, v]-B\left[x, v_{k}\right]\right| d x \\
& +\int_{\Omega}|z|\left|F[x, v]-F\left[x, v_{k}\right]\right| d x .
\end{aligned}
$$

We now give estimates for the integrals on the right hand side of (3.21). Using Lemma 2.2 with $\varepsilon>0$, we obtain ( $\sum$ negates implied sum)

$$
\begin{aligned}
& \text { (3.22) } \int_{\Omega}\left|\mathrm{z}_{\mathrm{x}}\right|\left|\mathrm{B}[\mathrm{x}, \mathrm{V}]-\mathrm{B}\left[\mathrm{x}, \mathrm{v}_{\mathrm{k}}\right]\right| \mathrm{dx} \leq \varepsilon \int_{\Omega}\left|\mathrm{z}_{\mathrm{x}}\right|^{2} \mathrm{dx} \\
& +(4 \varepsilon)^{-1} \sum_{\ell=1}^{n} \sum_{i=1}^{N} \int_{\Omega}\left|B_{i}^{l}[x, v]-B_{i}^{\ell}\left[x, v_{k}\right]\right|^{2} d x
\end{aligned}
$$

and

$$
\text { (3.23) } \begin{aligned}
& \int_{\Omega}|\mathrm{z}|\left|\mathrm{F}[\mathrm{x}, \mathrm{V}]-\mathrm{F}\left[\mathrm{x}, \mathrm{v}_{\mathrm{k}}\right]\right| \mathrm{dx} \leq \varepsilon \int_{\Omega}|\mathrm{z}|^{2} \mathrm{dx} \\
&+(4 \varepsilon)^{-1} \sum_{i=1}^{N} \int_{\Omega}\left|\mathrm{F}_{1}[\mathrm{x}, \mathrm{V}]-\mathrm{F}_{\mathrm{i}}\left[\mathrm{x}, \mathrm{v}_{\mathrm{k}}\right]\right|^{2} \mathrm{dx}
\end{aligned}
$$




$$
\begin{aligned}
& \leq \mathrm{b}^{2} \varepsilon \int_{\Omega}\left|\mathrm{z}_{\mathrm{x}}\right|^{2} \mathrm{dx} \\
& \quad+(4 \varepsilon)^{-1} \sum_{i=1}^{N} \int_{\Omega}\left|\mathrm{F}_{i}[\mathrm{x}, \mathrm{V}]-\mathrm{F}_{i}\left[\mathrm{x}, \mathrm{v}_{\mathrm{k}}\right]\right|^{2} \mathrm{dx}
\end{aligned}
$$

where $b$ is from (2.7) in the last inequality. The other term in (3.21) yields the estimate

$$
\int_{\Omega}\left|z_{x}\right|\left|C[x, v]-c\left[x, v_{k}\right]\right| \cdot\left|w_{x}\right| d x
$$$$
\leq \varepsilon \int_{\Omega}\left|\mathrm{z}_{\mathrm{x}}\right|^{2} \mathrm{dx}
$$$$
+(4 \varepsilon)^{-1} \sum_{i=1}^{N} \sum_{j=1}^{N} \sum_{\ell=1}^{n} \sum_{m=1}^{n} \int_{\Omega}\left|C_{i j}^{l m}[x, v]-c_{i j}^{l m}\left[x, v_{k}\right]\right|^{2}\left|w_{x}\right|^{2} d x .
$$

Choose $\varepsilon=\lambda\left[2\left(2+b^{2}\right)\right]^{-1}$ in the above inequalities. With this choice of $\varepsilon$, we may combine inequalities (3.21) through (3.24) to get

$$
\begin{aligned}
& || \Phi(\mathrm{V})-\Phi\left(\mathrm{V}_{\mathrm{k}}\right) \|_{2, \Omega} \\
& \left.\leq \quad\left[\left(2+\mathrm{b}^{2}\right) / 2 \lambda\right]\right]^{1 / 2}|| \mathrm{F}[\cdot, \mathrm{V}(\cdot)]-\mathrm{F}\left[\cdot, \mathrm{V}_{\mathrm{k}}(\cdot)\right] \|_{2, \Omega} \\
& +|| \mathrm{B}[\cdot, \mathrm{V}(\cdot)]-\left.\mathrm{B}\left[\cdot, \mathrm{v}_{\mathrm{k}}(\cdot)\right]\right|_{2, \Omega} \\
& +\left\|\left|\mathrm{C}[\cdot, \mathrm{V}(\cdot)]-\mathrm{C}\left[\cdot, \mathrm{V}_{\mathrm{k}}(\cdot)\right]\right|\left|\mathrm{w}_{\mathrm{x}}\right|\right\|_{2, \Omega} .
\end{aligned}
$$

The first two terms on the right hand side of (3.25) converge to zero as $k$ approaches $\infty$ by the continuity of $B$ and F given in Assumption 3.1. Thus, it suffices to show that the last term in (3.25) converges to zero. To do this, we let $\varepsilon>0$ be given and choose $U_{K}(\varepsilon) \varepsilon C_{0}^{\infty}(\Omega)$ such that 
$\left\|\mathrm{w}_{\mathrm{x}}-\mathrm{U}_{\mathrm{K}(\varepsilon)_{\mathrm{X}}}\right\|_{2, \Omega}<\varepsilon /\left(4 \mathrm{M}_{\mathrm{C}}\right)$ where $\mathrm{M}_{\mathrm{C}}$ is from Assumption 3.1. This yields

$$
\begin{aligned}
& \left.||\left|c[\cdot, v(\cdot)]-C\left[\cdot, v_{k}(\cdot)\right]\right|\left|w_{x}\right|\right|_{2, \Omega} \\
& \leq\left.||\left|C[\cdot, V(\cdot)]-C\left[\cdot, \mathrm{v}_{\mathrm{k}}(\cdot)\right]\right|\left|\mathrm{W}_{\mathrm{x}}-\mathrm{U}_{\mathrm{K}(\varepsilon)_{\mathrm{x}}}\right|\right|_{2, \Omega} \\
& +\left.||\left|C[\cdot, V(\cdot)]-C\left[\cdot, v_{k}(\cdot)\right]\right|\left|U_{K(\varepsilon)_{x}}\right|\right|_{2, \Omega} \\
& \leq 2 M_{C}|| W_{X}-U_{K}(\varepsilon)_{x} \|_{2, \Omega} \\
& +\sup _{x \varepsilon \Omega}\left|U_{K(\varepsilon)}(x)\right| \\
& \times|| c[\cdot, v(\cdot)]-c\left[\cdot, v_{k}(\cdot)\right] \|_{2, \Omega} \\
& <\varepsilon / 2+\mathrm{M}_{\mathrm{K}(\varepsilon)}\left\|\mathrm{C}[\cdot, \mathrm{V}(\cdot)]-\mathrm{C}\left[\cdot, \mathrm{v}_{\mathrm{k}}(\cdot)\right]\right\|_{2, \Omega}
\end{aligned}
$$

where $M_{K(\varepsilon)} \equiv \sup _{x \varepsilon \Omega}\left|U_{K(\varepsilon)}(x)\right|$. Now, since $c_{i j}^{l m}$ is

continuous from $\mathrm{L}_{2}(\Omega)$ into $\mathrm{L}_{2}(\Omega)$ from Assumption 3.1, there exists a positive integer $N(K(\varepsilon)$ ) depending only on $K(\varepsilon)$ such that $k>N(K(\varepsilon)$ ) implies

$$
{ }_{\mathrm{K}(\varepsilon)}|| C[\cdot, \mathrm{V}(\cdot)]-\mathrm{C}\left[\cdot, \mathrm{V}_{\mathrm{k}}(\cdot)\right] \|_{2, \Omega}<\varepsilon / 2 \text {. }
$$

Combining (3.26) and (3.27), we obtain the desired result, and the proof is complete.

LEMMA 3.2. The mapping $\Phi: \mathrm{L}_{2}(\Omega) \rightarrow \mathrm{L}_{2}(\Omega)$ is a compact mapping whenever Assumption 3.1 is valid.

PROOF. Suppose $A$ is a bounded set in $L_{2}(\Omega)$. Thus, there exists a constant $M_{1}$ such that $A$ is contained in the 
set $\left\{U \in L_{2}(\Omega)|||U|_{2, \Omega} \leq M_{1}\right\}$. We wish to show that $\Phi(A)$ is bounded in $H_{0}^{l}(\Omega)$. For $V \in A$, we put $z=W \equiv \Phi(V)$ in (3.6). With the use of Assumption 3.1, we get (3.28) $\lambda \int_{\Omega}\left|\mathrm{w}_{\mathrm{x}}\right|^{2} \mathrm{~d} \mathrm{x} \leq \int_{\Omega}\left|\mathrm{w}_{\mathrm{x}}\right||\mathrm{B}[\mathrm{x}, \mathrm{V}]| \mathrm{dx}+\int_{\Omega}|\mathrm{w}||\mathrm{F}[\mathrm{x}, \mathrm{v}]| \mathrm{dx}$. Lemma 2.2 now implies

$$
\text { (3.29) } \begin{aligned}
\lambda \int_{\Omega}\left|\mathrm{w}_{\mathrm{x}}\right|^{2} \mathrm{dx} & \leq \varepsilon \int_{\Omega}\left|\mathrm{w}_{\mathrm{x}}\right|^{2} \mathrm{~d} \mathrm{x}+(4 \varepsilon)^{-1} \int_{\Omega}|\mathrm{B}[\mathrm{x}, \mathrm{V}]|^{2} \mathrm{dx} \\
& +\varepsilon \int_{\Omega}|\mathrm{W}|^{2} \mathrm{~d} \mathrm{x}+(4 \varepsilon)^{-1} \int_{\Omega}|\mathrm{F}[\mathrm{x}, \mathrm{V}]|^{2} \mathrm{~d} \mathbf{x}
\end{aligned}
$$

for any $\varepsilon>0$. We now use (2.6) with $p=2$ and Assumption 3.1 to obtain

$$
\text { (3.30) } \begin{aligned}
\lambda \int_{\Omega}\left|\mathrm{w}_{\mathbf{x}}\right|^{2} \mathrm{dx} \leq & \varepsilon\left(1+\mathrm{b}^{2}\right) \int_{\Omega}\left|\mathrm{w}_{\mathbf{x}}\right|^{2} \mathrm{dx} \\
& +(4 \varepsilon)^{-1}\left[\left(\varepsilon_{0}+\varepsilon_{1}\right) \int_{\Omega}|\mathrm{v}|^{2} \mathrm{dx}\right. \\
& \left.+\left(\mathrm{M}_{\mathrm{F}}+\mathrm{M}_{\mathrm{B}}\right) \text { mes } \Omega\right]
\end{aligned}
$$

where $b$ is from (2.7). We now put $\varepsilon=\lambda\left[2\left(1+b^{2}\right)\right]^{-1}$ in (3.30) and simplify to get

$$
\begin{aligned}
\int_{\Omega}\left|W_{x}\right|^{2} d x \leq & \lambda^{-2}\left(1+b^{2}\right)\left[\left(\varepsilon_{0}+\varepsilon_{1}\right) \int_{\Omega}|v|^{2} d x\right. \\
& \left.+\left(M_{F}+M_{B}\right) \operatorname{mes} \Omega\right] .
\end{aligned}
$$

Corollary 2.1 and (3.31) now imply

$$
\begin{aligned}
|| w||_{0, \Omega}^{2} & =\left\|\left.\Phi(V)\right|_{0, \Omega} ^{2} \leq\left(1+b^{2}\right)|| w_{x}\right\|_{2, \Omega}^{2} \\
& \leq \lambda^{-2}\left(1+b^{2}\right)^{2}\left[\left(\varepsilon_{0}+\varepsilon_{1}\right) \int_{\Omega}|v|^{2} d x\right.
\end{aligned}
$$




$$
\left.+\left(M_{F}+M_{B}\right) \text { mes } \Omega\right] \text {. }
$$

Since $V \in A$, we have $\|V\|_{2, \Omega}^{2} \leq M_{1}^{2}$. Substituting this into (3.32) yields

$$
\begin{aligned}
\|\Phi(V)\|_{0, \Omega \leq} \leq & {\left[\lambda ^ { - 2 } ( 1 + b ^ { 2 } ) ^ { 2 } \left\{\left(\varepsilon_{0}+\varepsilon_{1}\right) M_{1}^{2}\right.\right.} \\
& \left.\left.+\left(M_{F}+M_{B}\right) \text { mes } \Omega\right\}\right]^{1 / 2} .
\end{aligned}
$$

Thus, $\Phi(A)$ is a bounded set in $H_{0}^{1}(\Omega)$ whenever $A$ is a bounded set in $\mathrm{L}_{2}(\Omega)$. Theorem 2.1 implies $\Phi(A)$ is relatively compact in $L_{2}(\Omega)$, and the proof is complete.

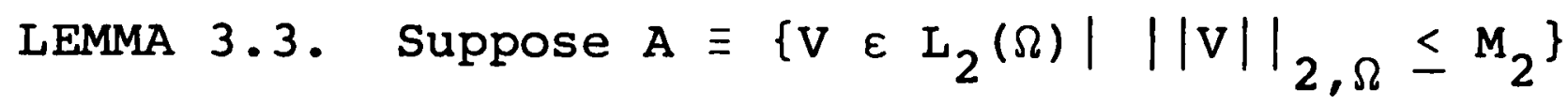
where

$$
M_{2}^{2} \equiv \frac{\left(M_{F}+M_{B}\right)\left(1+b^{2}\right)^{2} \text { mes } \Omega}{\lambda^{2}-\left(\varepsilon_{0}+\varepsilon_{1}\right)\left(1+b^{2}\right)^{2}}, b \text { is from }(2.7) \text {, }
$$

and $\varepsilon_{O}, \varepsilon_{1}, M_{F}$, and $M_{B}$ are from Assumption 3.1. Then $\Phi(A) \subset A$.

PROOF. Let $V$ be an element of A. From (3.32), we get

$$
\begin{aligned}
\|\Phi(V)\|_{0, \Omega}^{2} \leq & \lambda^{-2}\left(1+b^{2}\right)^{2}\left(\varepsilon_{o}+\varepsilon_{1}\right)\|v\|_{2, \Omega}^{2} \\
& +\lambda^{-2}\left(1+b^{2}\right)^{2}\left(M_{F}+M_{B}\right) \text { mes } \Omega .
\end{aligned}
$$

From the definition of $A$ and (3.35), we obtain

$$
\begin{aligned}
\|\Phi(V)\|_{0, \Omega}^{2} \leq & \lambda^{-2}\left(1+b^{2}\right)^{2}\left(\varepsilon_{0}+\varepsilon_{1}\right) M_{2}^{2} \\
& +\lambda^{-2}\left(1+b^{2}\right)^{2}\left(M_{F}+M_{B}\right) \text { mes } \Omega \\
\leq & M_{2}^{2}\left[\left(\varepsilon_{0}+\varepsilon_{1}\right)\left(1+b^{2}\right)^{2} \lambda^{-2}\right.
\end{aligned}
$$




$$
\begin{aligned}
& \left.+\lambda^{-2}\left\{\lambda^{2}-\left(\varepsilon_{0}+\varepsilon_{1}\right)\left(1+b^{2}\right)^{2}\right\}\right] \\
& =M_{2}^{2},
\end{aligned}
$$

and the proof is complete.

THEOREM 3.1. Suppose Assumption 3.1 is valid. Then the mapping $\Phi: \mathrm{L}_{2}(\Omega) \rightarrow \mathrm{L}_{2}(\Omega)$ has a fixed point, $\mathrm{U}$, in $\mathrm{L}_{2}(\Omega)$. Therefore, $\mathrm{U}$ is a solution of (1.1) and (1.2).

PROOF. Using Lemma 3.1 and Lemma 3.2 , we have that the mapping $\Phi: \mathrm{L}_{2}(\Omega) \rightarrow \mathrm{L}_{2}(\Omega)$ is compact. By Lemma 3.3, there is a closed bounded convex set $A$ in $L_{2}(\Omega)$ such that $\Phi(A)$ is contained in $A$. Theorem 2.2 implies $\Phi$ has a fixed point in $\mathrm{L}_{2}(\Omega)$, and the proof is complete.

We now change the conditions on $F$ and $B$ in Assumption 3.1 to allow $\varepsilon_{0}$ to be arbitrary and place a monotonicity type of constraint on $\mathrm{F}$.

ASSUMPTION 3.2. Suppose Assumption 3.1 is valid except for inequalities (3.3) and (3.4). We replace these inequalities with the following: There exists a constant $\eta$ and nonnegative constants $M$ and $\varepsilon_{I}$ such that

$$
\begin{aligned}
& \lambda-b^{2} \eta>0 \\
& \varepsilon_{1}<\left[b^{-1} \min \left\{\lambda, \lambda-b^{2} \eta\right\}\right]^{2} \\
& |F[x, U]|^{2} \leq M\left[1+|U|^{2}\right] \\
& \left(U^{i}-V^{i}\right)\left(F_{i}[x, U]-F_{i}[x, v]\right) \leq n|U-v|^{2}
\end{aligned}
$$

for all $U$ and $V$ in $E_{N}$ and $x$ in $\Omega$. 
In order to obtain a solution of (1.1) and (1.2) under Assumption 3.2, we first consider a different problem. We define a sequence of vector-valued functions $\left\{\mathrm{G}^{\mathrm{k}}\right\}_{\mathrm{k}=1}^{\infty}$ from $\Omega \times E_{N}$ into $E_{N}$ by $G_{i}^{k}[x, U]=\left(1+|U|^{2}\right)^{-1 / k} F_{i}[x, U]$ for $k=1,2, \ldots$ and $i=1,2, \ldots, N$. Let $v$ be a fixed element of $\mathrm{L}_{2}(\Omega)$ and $\mathrm{k}$ be an integer greater than 2 . We consider the problem of finding $\mathrm{W}_{k} \varepsilon \mathrm{H}_{0}^{l}(\Omega)$ such that

$$
\text { (3.41) } \begin{gathered}
\int_{\Omega} z_{, l}^{i} c_{i j}^{l m}[x, v] w_{k, m}^{j} d x+\int_{\Omega} z_{, l}^{i} B_{i}^{l}[x, v] d x \\
=\int_{\Omega} z^{i} G_{i}^{k}\left[x, w_{k}\right] d x
\end{gathered}
$$

for all $\mathrm{z}$ in $\mathrm{H}_{0}^{l}(\Omega)$. In order to do this, we observe that for each $U$ in $L_{2}(\Omega)$, there is a unique $g_{k}$ in $H_{0}^{l}(\Omega)$ such that

$$
\begin{aligned}
\int_{\Omega} z_{, l}^{i} & c_{i j}^{l m}[x, v] g_{k, m}^{i} d x+\int_{\Omega} z_{, l}^{i} B_{i}^{\ell}[x, v] d x \\
= & \int_{\Omega} z^{i} G_{i}^{k}[x, U] d x
\end{aligned}
$$

for all $\mathrm{z}$ in $\mathrm{H}_{0}^{l}(\Omega)$. The proof of this is identical to that of Proposition 3.3 and therefore omitted. This process defines a mapping $\mathrm{f}_{\mathrm{k}}: \mathrm{L}_{2}(\Omega) \rightarrow \mathrm{H}_{0}^{1}(\Omega)$ with $\mathrm{g}_{\mathrm{k}}=\mathrm{f}_{\mathrm{k}}(\mathrm{U})$. As in the previous existence argument, we shall show that $f_{k}$ has a fixed point in $\mathrm{L}_{2}(\Omega)$. This will yield a solution, $\mathrm{W}_{\mathrm{k}}$, to (3.41) for each $\mathrm{V}$ in $\mathrm{L}_{2}(\Omega)$ and each $\mathrm{k}=3,4, \ldots$ We now prove a series of lemmas concerning the mapping $f_{k}$.

LEMMA 3.4. The mapping $\mathrm{f}_{\mathrm{k}}: \mathrm{L}_{2}(\Omega) \rightarrow \mathrm{L}_{2}(\Omega)$ is continuous whenever Assumption 3.2 is valid. 
PROOF. Suppose $\left\{U_{\alpha}\right\}_{\alpha=1}^{\infty}$ is a sequence of functions in $\mathrm{L}_{2}(\Omega)$ such that $\mathrm{U}_{\alpha} \rightarrow \mathrm{U}$ in $\mathrm{L}_{2}(\Omega)$ as $\alpha \rightarrow \infty$. It suffices to show $\|\left.\left[f_{k}\left(U_{\alpha}\right)-f_{k}(U)\right]_{x}\right|_{2, \Omega} \rightarrow 0$ as $\alpha \rightarrow \infty$ because of Lemma 2.1. Using the corresponding forms of (3.42) for $f_{k}(U)$ and and $f_{k}\left(U_{\alpha}\right)$, we get

(3.43) $\int_{\Omega} z^{i}, l C_{i j}^{l m}[x, V]\left(f_{k}^{i}\left(U_{\alpha}\right)-f_{k}^{i}(U)\right), m d x$

$$
=\int_{\Omega} z^{i}\left(G_{i}^{k}\left[x, U_{\alpha}\right]-G_{i}^{k}[x, U]\right) d x
$$

for all $z$ in $H_{0}^{l}(\Omega)$. Let $z=f_{k}\left(U_{\alpha}\right)-f_{k}(U)$ in (3.43) and use Assumption 3.2, Lemma 2.1, and Lemma 2.2 to obtain

$$
\begin{aligned}
& \lambda \int_{\Omega}\left|\mathrm{z}_{\mathrm{x}}\right|^{2} \mathrm{dx} \leq \int_{\Omega}|\mathrm{z}|\left|\mathrm{G}^{\mathrm{k}}\left[\mathrm{x}, \mathrm{U}_{\alpha}\right]-\mathrm{G}^{\mathrm{k}}[\mathrm{x}, \mathrm{U}]\right| \mathrm{dx} \\
& \leq \varepsilon \int_{\Omega}|\mathrm{z}|^{2} \mathrm{dx} \\
& \quad+(4 \varepsilon)^{-1} \sum_{i=1}^{N} \int_{\Omega}\left|\mathrm{G}_{i}^{\mathrm{k}}\left[\mathrm{x}, \mathrm{U}_{\alpha}\right]-G_{i}^{\mathrm{k}}[\mathrm{x}, \mathrm{U}]\right|^{2} \mathrm{dx} \\
& \leq \varepsilon \mathrm{b}^{2} \int_{\Omega}\left|\mathrm{z}_{\mathrm{x}}\right|^{2} \mathrm{dx} \\
& \quad+(4 \varepsilon)^{-1} \sum_{i=1}^{N} \int_{\Omega}\left|G_{i}^{\mathrm{k}}\left[\mathrm{x}, \mathrm{U}_{\alpha}\right]-\mathrm{G}_{i}^{\mathrm{k}}[\mathrm{x}, \mathrm{U}]\right|^{2} \mathrm{dx}
\end{aligned}
$$

where $b$ is from (2.7). In (3.44), let $\varepsilon=\lambda / 2 b^{2}$ and simplify to get

$$
\text { (3.45) } \begin{aligned}
\|\left[f_{k}\left(U_{\alpha}\right)\right. & \left.-f_{k}(U)\right]_{x} \|\left.\right|_{2, \Omega} \\
\leq & (b / \lambda)\left\|G^{k}\left[\cdot, U_{\alpha}(\cdot)\right]-G^{k}[\cdot, U(\cdot)]\right\|_{2, \Omega} \cdot
\end{aligned}
$$


Since $F$ is a continuous mapping from $L_{2}(\Omega)$ into $L_{2}(\Omega)$, it is clear that $G^{k}$ also has this property. Thus, letting $\alpha \rightarrow \infty$, we get $\left\|\mathrm{G}^{\mathrm{k}}\left[\cdot, \mathrm{U}_{\alpha}(\cdot)\right]-\mathrm{G}^{\mathrm{k}}[\cdot, \mathrm{U}(\cdot)]\right\|_{2, \Omega} \rightarrow 0$, and the proof is complete.

LEMMA 3.5. The mapping $\mathrm{f}_{\mathrm{k}}: \mathrm{L}_{2}(\Omega) \rightarrow \mathrm{L}_{2}(\Omega)$ is a compact mapping whenever Assumption 3.2 is valid.

PROOF. In view of Theorem 2.1, it suffices to show that if $A$ is a bounded set in $L_{2}(\Omega)$, then $f_{k}(A)$ is a bounded set in $H_{0}^{l}(\Omega)$. Thus, suppose $A \subset\left\{U \in L_{2}(\Omega)|||U|_{2, \Omega} \leq L\right\}$ for some positive number $L$. If $U \in A$, then letting $\mathrm{z}=\mathrm{f}_{\mathrm{k}}(\mathrm{U})=\mathrm{g}_{\mathrm{k}}$ in (3.42) and using Assumption 3.2 yields

$$
\begin{aligned}
& \lambda \int_{\Omega}\left|z_{x}\right|^{2} \mathrm{dx} \leq \int_{\Omega}\left|\mathrm{z}_{\mathrm{x}}\right||\mathrm{B}[\mathrm{x}, \mathrm{V}]| \mathrm{dx} \\
&+\int_{\Omega}|\mathrm{z}|\left|\mathrm{G}^{\mathrm{k}}[\mathrm{x}, \mathrm{U}]\right| \mathrm{dx} .
\end{aligned}
$$

By using Lemma 2.1 and Lemma 2.2 as well as Assumption 3.2, we get

$$
\text { (3.47) } \begin{aligned}
\lambda & \int_{\Omega}\left|\mathrm{z}_{\mathrm{x}}\right|^{2} \mathrm{dx} \leq \varepsilon \int_{\Omega}\left|\mathrm{z}_{\mathrm{x}}\right|^{2} \mathrm{dx}+\varepsilon \int_{\Omega}|\mathrm{z}|^{2} \mathrm{dx} \\
& +(4 \varepsilon)^{-1} \int_{\Omega}|\mathrm{B}[\mathrm{x}, \mathrm{V}]|^{2} \mathrm{dx} \\
& +(4 \varepsilon)^{-1} \sum_{i=1}^{N} \int_{\Omega}\left|\mathrm{G}_{\mathrm{i}}^{\mathrm{k}}[\mathrm{x}, \mathrm{U}]\right|^{2} \mathrm{dx} \\
& \leq \varepsilon\left(1+\mathrm{b}^{2}\right) \int_{\Omega}\left|\mathrm{z}_{\mathrm{x}}\right|^{2} \mathrm{dx} \\
& +\mathrm{M}_{\varepsilon}+\mathrm{M}(4 \varepsilon)^{-1} \int_{S_{6}}\left(1+|\mathrm{U}|^{2}\right)^{1-2 / \mathrm{k}} \mathrm{dx}
\end{aligned}
$$


where $M_{\varepsilon} \equiv(4 \varepsilon)^{-1} \int_{\Omega}|B[x, V]|^{2} d x$ and $b$ is from (2.7). Let $\varepsilon=\hat{\varepsilon}=\lambda\left[2\left(1+b^{2}\right)\right]^{-1}$ in $(3.47)$ and simplify to obtain

(3.48) $\quad|| z_{x} \|_{2, \Omega}^{2}$

$$
\begin{aligned}
& \leq 2 M_{\hat{\varepsilon}} / \lambda+M\left(1+b^{2}\right) /\left(\lambda^{2}\right) \int_{\Omega}\left(1+|U|^{2}\right)^{1-2 / k} d x \\
& \leq 2 M_{\hat{\varepsilon}} / \lambda+M\left(1+b^{2}\right) /\left(\lambda^{2}\right) \int_{\Omega}\left(1+|U|^{2}\right) d x \\
& \leq 2 M_{\hat{\varepsilon}} / \lambda+\left[M\left(1+b^{2}\right) /\left(\lambda^{2}\right)\right]\left(\text { mes } \Omega+L^{2}\right) \equiv \hat{L} .
\end{aligned}
$$

This inequality holds for all U $\varepsilon$ A, and now Corollary 2.1 gives

(3.49) $\left.\left.|| z\right|_{0, \Omega} \equiv|| f_{k}(U)\left\|_{0, \Omega} \leq\left(1+b^{2}\right)^{1 / 2}\right\|\left[f_{k}(U)\right]_{x}\right|_{2, \Omega}$

$$
\leq\left[\left(1+b^{2}\right) \hat{L}\right]^{1 / 2}
$$

for all $U \in A$, and the proof is complete.

LEMMA 3.6. Suppose $A_{k}=\left\{U \in L_{2}(\Omega) \mid\|U\|_{2, \Omega \leq \tilde{M}_{k}}\right\}$ where

$$
\begin{aligned}
\tilde{M}_{k}^{2} \equiv & 2\left(1+b^{2}\right)\left\{2 \hat{M} / \lambda+(2 P / k)\left[2(k-2)\left(1+b^{2}\right) / k\right](k-2) / 2\right. \\
& \left.+(\operatorname{mes} \Omega) /\left[2\left(1+b^{2}\right)\right]\right\}
\end{aligned}
$$

with $\hat{M} \equiv\left[\left(1+b^{2}\right) /(2 \lambda)\right] \int_{\Omega}|B[x, V]|^{2} d x, b$ is from $(2.7)$, and $P \equiv\left[M\left(1+b^{2}\right) /\left(\lambda^{2}\right)\right]^{k / 2}$. Then $f_{k}\left(A_{k}\right) \subset A_{k}$. 
PROOF. Let $U \in A_{k}$. Using the first inequality of (3.48) and Lemma 2.2 (with $\lambda=1-2 / k, a=1+|u|^{2}, k>2$ ) yields

$$
\text { (3.50) } \begin{aligned}
\left\|\left[\mathrm{f}_{\mathrm{k}}(\mathrm{U})\right]_{\mathrm{x}}\right\|_{2, \Omega \leq} \leq & \left\{2 \hat{\mathrm{M}} / \lambda+\varepsilon \int_{\Omega}\left(1+|\mathrm{U}|^{2}\right) \mathrm{dx}\right. \\
& +(2 / \mathrm{k})[(\mathrm{k}-2) / \varepsilon \mathrm{k}](\mathrm{k}-2) / 2 \mathrm{P}\}^{1 / 2} \\
= & \varepsilon \int_{\Omega}|\mathrm{U}|^{2} \mathrm{dx}+\mathrm{K}(\varepsilon, \mathrm{k})
\end{aligned}
$$

where $\varepsilon>0, \hat{M}=M_{\hat{\varepsilon}}$ of $(3.48)$, and

$\mathrm{K}(\varepsilon, \mathrm{k}) \equiv(2 \hat{\mathrm{M}} / \lambda)+(2 / \mathrm{k})[(\mathrm{k}-2) / \varepsilon \mathrm{k}](\mathrm{k}-2) / 2 \mathrm{p}+\varepsilon($ mes $\Omega)$.

Thus, $U \in A_{k}$ implies

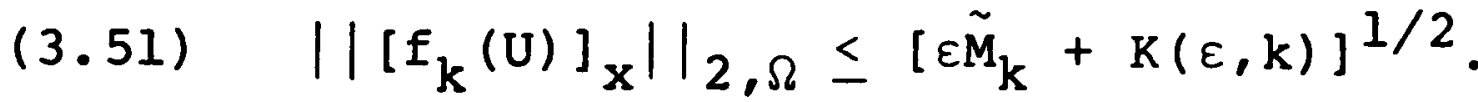

Corollary 2.1 along with $(3.51)$ gives

$$
\left\|f_{k}(U)\right\|_{0, \Omega} \leq\left\{\left(1+b^{2}\right)\left[\varepsilon \tilde{M}_{k}+K(\varepsilon, k)\right]\right\}^{1 / 2} .
$$

Put $\varepsilon=\left[2\left(1+b^{2}\right)\right]^{-1}$ in $(3.52)$ and get

(3.53)

$$
\begin{aligned}
\left\|f_{k}(U)\right\|_{0, \Omega \leq} \leq & \left\{( 1 + b ^ { 2 } ) \left[\left\{2\left(1+b^{2}\right)\right\}^{-1} \tilde{M}_{k}^{2}\right.\right. \\
& \left.\left.+\left[2\left(1+b^{2}\right)\right]^{-1} \tilde{M}_{k}^{2}\right]\right\}^{1 / 2} \\
= & \tilde{M}_{k}
\end{aligned}
$$

where we have used the identity $[K(\varepsilon, k)]^{2}=\tilde{M}_{k}^{2} /\left[2\left(1+b^{2}\right)\right]$ for $\varepsilon=\left[2\left(1+b^{2}\right)\right]^{-1}$, and the proof is complete.

THEOREM 3.2. Suppose Assumption 3.2 is valid. Then 
the mapping $\mathrm{f}_{\mathrm{k}}: \mathrm{L}_{2}(\Omega) \rightarrow \mathrm{L}_{2}(\Omega)$ has a fixed point in $\mathrm{L}_{2}(\Omega)$. Therefore, (3.41) has at least one solution for each $k \geq 3$ and each $\mathrm{V}$ in $\mathrm{L}_{2}(\Omega)$.

PROOF. Lemma 3.4 and Lemma 3.5 imply the mapping $f_{k}: L_{2}(\Omega) \rightarrow L_{2}(\Omega)$ is compact. By Lemma 3.6 , there is a closed convex bounded set $A_{k}$ such that $f_{k}\left(A_{k}\right) \subset A_{k}$. Therefore, Theorem 2.2 implies $f_{k}$ has a fixed point, and the proof is complete.

We now show that the sequence $\left\{w_{k}\right\}_{k=1}^{\infty}$ of solutions to (3.41) has a convergent subsequence in $H_{0}^{l}(\Omega)$. First, we need a preliminary lemma.

LEMMA 3.7. For each $V$ in $L_{2}(\Omega)$, there is a positive constant $\mathrm{L}$ depending on $\mathrm{V}$ but independent of $\mathrm{k}$ such that $\left.|| w_{k}\right|_{0, \Omega} \leq L$ for all $k \geq 3$.

PROOF. In (3.41), let $\mathrm{z}=\mathrm{W}_{\mathrm{k}}$ and use Assumption 3.2 to get

$$
\text { (3.54) } \begin{aligned}
\lambda \int_{\Omega}\left|\mathrm{z}_{\mathrm{x}}\right|^{2} \mathrm{dx} \leq & \int_{\Omega}\left|\mathrm{z}_{\mathrm{x}}\right||\mathrm{B}[\mathrm{x}, \mathrm{V}]| \mathrm{dx} \\
& +\sum_{i=1}^{N} \int_{\Omega} \mathrm{w}_{\mathrm{k}}^{\mathrm{i}} \mathrm{G}_{\mathrm{i}}^{\mathrm{k}}\left[\mathrm{x}, \mathrm{w}_{\mathrm{k}}\right] \mathrm{dx} .
\end{aligned}
$$

With the use of Lemma 2.2 , we have

(3.55) $\int_{\Omega}\left|\mathrm{z}_{\mathrm{x}}\right||\mathrm{B}[\mathrm{x}, \mathrm{V}]| \mathrm{dx} \leq \varepsilon \int_{\Omega}\left|\mathrm{z}_{\mathrm{x}}\right|^{2} \mathrm{dx}$

$$
+(4 \varepsilon)^{-1} \int_{\Omega}|B[x, v]|^{2} d x
$$


If $n \geq 0$ in (3.37), inequality (3.40) (with $U=W_{k}$ and $\mathrm{V}=0$ ) implies

$$
\text { (3.56) } \begin{aligned}
& \sum_{i=1}^{N} W_{k}^{i} G_{i}^{k}\left[x, w_{k}\right]=\sum_{i=1}^{N} w_{k}^{i}\left(1+\sum_{j=1}^{N}\left|w_{k}^{j}\right|^{2}\right)^{-1 / k} F_{i}\left[x, w_{k}\right] \\
\leq & {\left[1+\sum_{j=1}^{N}\left|w_{k}^{j}\right|^{2}\right]^{-1 / k}\left[\sum_{i=1}^{N} n\left|w_{k}^{i}\right|^{2}+\left|w_{k}\right||F[x, 0]|^{2}\right] } \\
\leq & {\left[1+\sum_{i=1}^{N}\left|w_{k}^{i}\right|^{2}\right]^{-1 / k}\left[(n+\varepsilon) \sum_{i=1}^{N}\left|w_{k}^{i}\right|^{2}+(4 \varepsilon)^{-1}|F[x, 0]|^{2}\right] } \\
& \leq(n+\varepsilon) \sum_{i=1}^{N}\left|w_{k}^{i}\right|^{2}+(4 \varepsilon)^{-1}|F[x, 0]|^{2} \\
& \equiv(n+\varepsilon)|z|^{2}+(4 \varepsilon)^{-1}|F[x, 0]|^{2} .
\end{aligned}
$$

Combining $(3.54),(3.55)$, and $(3.56)$ yields

$$
\text { (3.57) } \begin{aligned}
(\lambda-\varepsilon) & \int_{\Omega}\left|z_{x}\right|^{2} d x \leq(\eta+\varepsilon) \int_{\Omega}|z|^{2} d x \\
& +(4 \varepsilon)^{-1}\left[\int_{\Omega}|B[x, v]|^{2} d x+\int_{\Omega}|F[x, 0]|^{2} d x\right] .
\end{aligned}
$$

Lemma 2.1 and (3.57) imply

$$
\text { (3.58) } \begin{aligned}
{[\lambda-\varepsilon} & \left.-\mathrm{b}^{2}(n+\varepsilon)\right] \int_{\Omega}|\mathrm{z}|^{2} \mathrm{dx} \\
& +(4 \varepsilon)^{-1}\left[\int_{\Omega}|\mathrm{B}[\mathrm{x}, \mathrm{V}]|^{2} \mathrm{dx}+\int_{\Omega}|\mathrm{F}[\mathrm{x}, 0]|^{2} \mathrm{dx}\right] .
\end{aligned}
$$

We now choose $\varepsilon=\left(\lambda-b^{2} \eta\right)\left[2\left(1+b^{2}\right)\right]^{-1}$ in (3.58). Simplifying (3.58) yields 
(3.59) $\int_{\Omega}\left|z_{x}\right|^{2} d x \leq\left(1+b^{2}\right) /\left(\lambda-b^{2} \eta\right)^{2}\left[\int_{\Omega}|B[x, v]|^{2} d x\right.$

$$
\left.+\int_{\Omega}|F[x, 0]|^{2} d x\right] \equiv \hat{L} \text {. }
$$

Corollary 2.1 and (3.59) now yield

(3.60) $\quad|| w_{k} \|\left._{0, \Omega} \equiv|| z\right|_{0, \Omega} \leq\left[\left(1+b^{2}\right) \hat{L}\right]^{1 / 2}$.

If $\eta<0$ in (3.37), we have from (3.40) the inequality

(3.61) $\sum_{i=1}^{N} w_{k}^{i} G_{i}^{k}\left[x, w_{k}=\sum_{i=1}^{N}\left(1+\sum_{j=1}^{N}\left|w_{k}^{j}\right|^{2}\right)^{-1 / k} w_{k}^{i} F_{i}\left[x, w_{k}\right]\right.$

$$
\begin{aligned}
& \leq\left(1+\sum_{j=1}^{N}\left|w_{k}^{j}\right|^{2}\right)^{-1 / k}\left[n \sum_{i=1}^{N}\left|w_{k}^{i}\right|^{2}+\left|w_{k}\right||F[x, 0]|\right] \\
& \leq\left|w_{k}\right||F[x, 0]| \\
& \leq \varepsilon \sum_{i=1}^{N}\left|w_{k}^{i}\right|^{2}+(4 \varepsilon)^{-1}|F[x, 0]|^{2} \\
& \equiv \varepsilon|z|^{2}+(4 \varepsilon)^{-1}|F[x, 0]|^{2} .
\end{aligned}
$$

Combining $(3.54),(3.55)$, and (3.61) yields

$$
\text { (3.62) } \begin{aligned}
(\lambda-\varepsilon) & \int_{\Omega}\left|\mathrm{z}_{\mathrm{x}}\right|^{2} \mathrm{dx} \leq \varepsilon \int_{\Omega}|\mathrm{z}|^{2} \mathrm{dx} \\
& +(4 \varepsilon)^{-1}\left[\int_{\Omega}|\mathrm{B}[\mathrm{x}, \mathrm{V}]|^{2} \mathrm{dx}+\int_{\Omega}|\mathrm{F}[\mathrm{x}, 0]|^{2} \mathrm{dx}\right] .
\end{aligned}
$$

The analysis now follows as for $\eta \geq 0$ in (3.58), (3.59), 
and $(3.60)$ as if $\eta=0$ and $\varepsilon=\lambda\left[2\left(1+b^{2}\right)\right]^{-1}$ to get

$$
\begin{aligned}
& || w_{k} \|_{0, \Omega} \equiv|| z||_{0, \Omega} \\
& \leq\left[\left(1+b^{2}\right)^{1 / 2} / \lambda\right]\left[\int_{\Omega}|B[x, v]|^{2} \mathrm{dx}+\int_{\Omega}|F[x, 0]|^{2} \mathrm{dx}\right]^{1 / 2},
\end{aligned}
$$

and the proof is complete.

LEMMA 3.8. The sequence $\left\{\mathrm{w}_{\mathrm{k}}\right\}_{\mathrm{k}=3}^{\infty}$ has a subsequence $\left\{W_{k_{\nu}}\right\}_{\nu=1}^{\infty}$ which converges in $\mathrm{H}_{0}^{l}(\Omega)$.

PROOF. Lemma 3.7 along with Theorem 2.1 implies the existence of a subsequence $\left\{W_{k_{v}}\right\}_{\nu=1}^{\infty}$ which converges in $\mathrm{L}_{2}(\Omega)$. We now show that this subsequence is Cauchy in $H_{0}^{1}(\Omega)$. Thus suppose $W_{k_{\nu}}$ and $W_{k_{\alpha}}$ are elements of the subsequence. With the use of (3.41), we get

$$
\text { (3.64) } \begin{array}{rl}
\int_{\Omega} z_{, \ell}^{i} C_{i j}^{l m}[x, v]\left[w_{k_{\nu}}^{j}-w_{k_{\alpha}}^{j}\right], m & d x \\
& =\int_{\Omega} z^{i}\left[G_{i}^{k \nu}\left[x, w_{k_{\nu}}\right]-G_{i}^{k \alpha}\left[x, w_{k_{\alpha}}\right]\right] d x
\end{array}
$$

for all $\mathrm{z}$ in $\mathrm{H}_{0}^{l}(\Omega)$. We put $\mathrm{z}=\mathrm{W}_{\mathrm{k}_{v}}-\mathrm{W}_{\mathrm{k}_{\alpha}}$ in (3.64) and use Assumption 3.2 to obtain

$$
\text { (3.65) } \begin{aligned}
\lambda \sum_{i=1}^{N} \int_{\Omega}\left|\left[w_{k_{\nu}}^{i}-w_{k_{\alpha}}^{i}\right]\right|^{2} d x \\
\leq \sum_{i=1}^{N} \int_{\Omega}\left(w_{k_{\nu}}^{i}-w_{k_{\alpha}}^{i}\right)\left(G_{i}^{k} v\left[x, w_{k_{\nu}}\right]-G_{i}^{k}\left[x, w_{k_{\alpha}}\right]\right) d x
\end{aligned}
$$




$$
\begin{aligned}
& \leq \sum_{i=1}^{N} \int_{\Omega}\left|w_{k_{\nu}}^{i}-w_{k_{\alpha}}^{i}\right|^{2} d x \\
&+\sum_{i=1}^{N} \int_{\Omega}\left|G_{i}^{k_{\nu}}\left[x, w_{k_{\nu}}\right]-G_{i}^{k_{\alpha}}\left[x, w_{k_{\alpha}}\right]\right|^{2} d x .
\end{aligned}
$$

Therefore, to show that the subsequence $\left\{w_{k_{\nu}}\right\}_{\nu=1}^{\infty}$ is cauchy in $\mathrm{H}_{0}^{l}(\Omega)$, it suffices to show that the last integral on the right hand side of (3.65) approaches zero as $\nu$ and $\alpha$ approach $\infty$. To do this, it suffices to show

$$
\text { (3.66) } \lim _{\nu \rightarrow \infty} \sum_{i=1}^{N} \int_{\Omega}\left|G_{i}^{k_{\nu}}\left[x, w_{k_{\nu}}\right]-F_{i}\left[x, w_{k_{\nu}}\right]\right|^{2} d x=0
$$

since we already know $\left\{\mathrm{F}\left[\cdot, \mathrm{w}_{\mathrm{k}_{\nu}}(\cdot)\right]\right\}_{\nu=1}^{\infty}$ is Cauchy in $\mathrm{L}_{2}(\Omega)$. For simplicity in notation, we replace $w_{k_{v}}$ with $Y$ for the remainder of the proof. From the definition of $\mathrm{G}^{\mathrm{k}}$ and Assumption 3.2 , we obtain

$$
\text { (3.67) } \begin{aligned}
I & \equiv \sum_{i=1}^{N} \int_{\Omega}\left|G_{i}^{k \nu}[\mathrm{X}, \mathrm{Y}]-\mathrm{F}_{i}[\mathrm{X}, \mathrm{Y}]\right|^{2} \mathrm{dx} \\
& =\int_{\Omega}\left|\left[1+|\mathrm{Y}|^{2}\right]^{-1 / \mathrm{k} \nu} \mathrm{F}[\mathrm{X}, \mathrm{Y}]-\mathrm{F}[\mathrm{X}, \mathrm{Y}]\right|^{2} \mathrm{dx} \\
& \leq \int_{\Omega}|\mathrm{F}[\mathrm{X}, \mathrm{Y}]|^{2} \mid 1-\left(1+|\mathrm{Y}|^{2}\right)^{-1 /\left.\mathrm{k} \nu\right|^{2} \mathrm{dx}} \\
& \leq \mathrm{M} \int_{\Omega}\left(1+|\mathrm{Y}|^{2}\right)\left|1-\left(1+|\mathrm{Y}|^{2}\right)^{-1 / k \nu}\right|^{2} \mathrm{dx} \\
& \leq \mathrm{M} \int_{\Omega}(1+|\mathrm{Y}|)^{2}\left[\left(1+|\mathrm{Y}|^{2}\right)^{1 / \mathrm{K} \nu}-1\right]^{2} \mathrm{dx} .
\end{aligned}
$$


The last integral in (3.67) is finite by virtue of Lemma 2.1 whenever $\nu$ is large enough that $2+4 / k_{\nu}<2 n /(n-2)$ whenever $n \geq 3$. If $n=2$, it is finite for all $v$. Using that same lemma and Hölder's inequality in (3.67), we get

$$
\text { (3.68) } \begin{aligned}
& I \leq M\left[\int_{\Omega}(1+|Y|)^{2 n /(n-2)} d x\right](n-2) / n \\
&\left.\times\left(\int_{\Omega}\left[1+|Y|^{2}\right) 1 / k \nu-1\right]^{n} d x\right)^{2 / n} \\
& \leq 4 M\left[\int_{\Omega}\left(1+|Y|^{2 n /(n-2)}\right) d x\right]^{(n-2) / n} \times Q \\
& \leq 4 M\left[\text { mes } \Omega+|| Y x||_{2, \Omega}^{2 n /(n-2)}\right]^{(n-2) / n} \times Q \\
& \leq 4 M\left[\text { mes } \Omega+L^{n /(n-2)}\right]^{(n-2) / n} \times Q
\end{aligned}
$$

where $L$ is the bound for ||$w_{k} \|_{0, \Omega}$ given in Lemma 3.7 and $\left.Q \equiv\left(\int_{\Omega}\left[1+|Y|^{2}\right)^{1 / k \nu}-1\right]^{n} d x\right)^{2 / n}$. It remains for us to show that the integral $Q$ on the right hand side of (3.68) converges to zero as $v \rightarrow \infty$. To do this, we use the identity

$$
\begin{aligned}
& \int_{\Omega}\left\{\left(I+|\mathrm{Y}|^{2}\right)^{\left.1 / \mathrm{k}_{\nu}-1\right\}^{\mathrm{n}} \mathrm{dx}}\right. \\
& \left.=\sum_{\beta=0}^{\mathrm{n}}(-1)^{\beta} \mid \begin{array}{l}
\mathrm{n} \\
\beta
\end{array}\right) \int_{\Omega}\left(1+|\mathrm{Y}|^{2}\right)^{(\mathrm{n}-\beta) / \mathrm{k}_{\nu} \mathrm{dx}},
\end{aligned}
$$

and show 
(3.70) $\lim _{\nu \rightarrow \infty} \int_{\Omega}\left(1+|Y|^{2}\right)^{(n-\beta) / k_{\nu}} d x$

$$
\equiv \lim _{\nu \rightarrow \infty} \int_{\Omega}\left(1+\sum_{i=1}^{N}\left|w_{k_{\nu}}^{i}\right|^{2}\right)^{(n-\beta) / k_{\nu}} d x=\operatorname{mes} \Omega
$$

for all $0 \leq \beta \leq n$. We observe that (3.70) is trivial for $\beta=\mathrm{n}$ and consider only the cases $0 \leq \beta \leq \mathrm{n}-1$. Thus, for $k_{v} \geq \mathrm{n}+1,(3.70)$ yields the estimate

$$
\begin{aligned}
& \text { (3.71) mes } \Omega \leq \int_{\Omega}\left(1+|\mathrm{Y}|^{2}\right)(n-\beta) / \mathrm{k}_{\nu} \mathrm{dx} \\
& \leq \int_{\Omega}(1+|Y|)^{2(n-\beta) / k_{\nu}} \\
& \leq\left[\int_{\Omega} 1 d x\right)^{1-\left[(n-\beta) / k_{v}\right]} \\
& \times\left(\int_{\Omega}[1+|Y|]^{2} d x\right)^{(n-\beta) / k_{\nu}} \\
& \leq(\operatorname{mes} \Omega)^{1-\left[(n-\beta) / k_{\nu}\right]}\left[2\left(\operatorname{mes} \Omega+L^{2}\right)\right]^{(n-\beta) / k_{\nu}}
\end{aligned}
$$

where $L$ is from Lemma 3.7. Since the term on the right hand side of (3.71) approaches mes $\Omega$ as $\nu$ approaches $\infty$, we obtain $(3.70)$. Now (3.70) and (3.69) yield $\left(Y \equiv W_{k_{V}}\right)$

$$
\text { (3.72) } \begin{aligned}
\lim _{\nu \rightarrow \infty} \int_{\Omega}\left|1-\left(1+\sum_{j=1}^{N}\left|w_{k_{\nu}}^{j}\right|^{2}\right)^{1 / k_{\nu}}\right|^{n} \mathrm{~d} \mathbf{x} \\
=\sum_{\beta=0}^{n}(-1)^{\beta}\left|\begin{array}{l}
\mathrm{n} \\
\beta
\end{array}\right| \text { mes } \Omega=0,
\end{aligned}
$$

and the proof is complete. 
THEOREM 3.3. Let $V \in \mathrm{L}_{2}(\Omega)$, and suppose Assumption 3.2 is valid. Then there is a unique $W$ in $H_{0}^{l}(\Omega)$ such that

(3.73) $\int_{\Omega} \mathrm{z}_{, \ell}^{i} C_{i j}^{l m}[\mathrm{x}, \mathrm{v}] \mathrm{w}_{, \mathrm{m}}^{j} \mathrm{dx}+\int_{\Omega} \mathrm{z}_{, \ell}^{i} \mathrm{~B}_{i}^{\ell}[\mathrm{x}, \mathrm{v}] \mathrm{dx}$

$$
=\int_{\Omega} z^{i} F_{i}[x, W] d x
$$

for all $\mathrm{z}$ in $\mathrm{H}_{0}^{1}(\Omega)$.

PROOF. From Theorem 3.2 , there is a sequence $\left\{w_{k}\right\}_{k=3}^{\infty}$ contained in $\mathrm{H}_{0}^{l}(\Omega)$ which satisfies the equence of equations given by (3.41). Lemma 3.8 implies the existence of a subsequence $\left\{\mathrm{w}_{\mathrm{k}_{v}}\right\}_{\nu=1}^{\infty}$ of $\left\{\mathrm{w}_{\mathrm{k}}\right\}_{\mathrm{k}=3}^{\infty}$ which converges to a function $\mathrm{W}$ in $\mathrm{H}_{0}^{l}(\Omega)$. We observe that

(3.74) $\int_{\Omega} z_{, l}^{i} c_{i j}^{\ell m}[x, v] w_{k_{\nu}, m}^{j} d x \rightarrow \int_{\Omega} z_{, l}^{i} c_{i j}^{l m}[x, v] w_{, m}^{j} d x$ as $v \rightarrow \infty$ since $W_{k,, m} \rightarrow W_{, m}$ in $I_{2}(\Omega)$. Also, the continuity of $F$ given in Assumption 3.2 along with (3.66) yields

(3.75) $\lim _{\nu \rightarrow \infty} \sum_{i=1}^{N} \int_{\Omega}\left|G_{i}^{k_{\nu}}\left[x, w_{k \nu}\right]-F_{i}[x, w]\right|^{2} d x=0$, and this implies

(3.76) $\lim _{\nu \rightarrow \infty} \int_{\Omega} z^{i} G_{i}^{k_{\nu}}\left[x, w_{k_{\nu}}\right] d x=\int_{\Omega} z^{i} F_{i}[x, w] d x$ for all $\mathrm{z}$ in $\mathrm{H}_{0}^{1}(\Omega)$, and thus, $\mathrm{W}$ is a solution of (3.73). 
For the uniqueness, suppose $W$ and $\tilde{W}$ are solutions of (3.73) corresponding to some $V$ in $L_{2}(\Omega)$. Then

(3.77) $\int_{\Omega} z_{, l}^{i} c_{i j}^{l m}[x, v]\left(w^{j}-\tilde{w}^{j}\right), m d x$

$$
=\int_{\Omega} z^{i}\left(F_{i}[x, W]-F_{i}[x, \tilde{w}]\right) d x
$$

for all $\mathrm{z}$ in $\mathrm{H}_{0}^{1}(\Omega)$. Put $\mathrm{Z}=\mathrm{W}-\tilde{\mathrm{W}}$ in (3.77), and use Assumption 3.2 to get

$$
\lambda \int_{\Omega}|[w-\tilde{w}]|^{2} d x \leq n \int_{\Omega}|w-\tilde{w}|^{2} d x .
$$

If $n \leq 0$, then $\left\|[w-\tilde{w}]_{x}\right\|_{2, \Omega}^{2} \leq 0$ and $w=\tilde{w}$. If $n>0$, we apply Lemma 2.1 with $p=2$ and use (3.78) to get

$$
\lambda \int_{\Omega}\left|[w-\tilde{w}]{ }_{x}\right|^{2} d x \leq n b^{2} \int_{\Omega}|[w-\tilde{w}] x|^{2} d x
$$

which implies

$$
\left.\left(\lambda-b^{2} n\right) \int_{\Omega}|[w-\tilde{w}]|_{x}\right|^{2} d x \leq 0 .
$$

By virtue of (3.37), we obtain $W=\tilde{W}$, and the proof is complete.

With the use of Theorem 3.3, we can define a mapping $h: L_{2}(\Omega) \rightarrow H_{0}^{l}(\Omega)$ by $h(V)=W$ so that $W$ is the solution of (3.73) whenever $\mathrm{V}$ is an element of $\mathrm{L}_{2}(\Omega)$. We can now prove the existence of a solution to (1.1) and (1.2) under Assumption 3.2 by showing that the mapping $h$ has a fixed point. Once again, we prove a series of lemmas concerning the mapping $h$. 
LEMMA 3.9. The mapping $h: I_{2}(\Omega) \rightarrow L_{2}(\Omega)$ is continuous whenever As sumption 3.2 is valid.

PROOF. Let $\left\{\mathrm{V}_{\mathrm{k}}\right\}_{\mathrm{k}=1}^{\infty}$ be a sequence of functions in $\mathrm{L}_{2}(\Omega)$ such that $\mathrm{v}_{\mathrm{k}}+\mathrm{V}$ in $\mathrm{L}_{2}(\Omega)$ as $\mathrm{k} \rightarrow \infty$. In view of Lemma 2.1, it suffices to show $\|\left[w_{k}-w_{x} \|_{2, \Omega} \rightarrow 0\right.$ where $w_{k} \equiv h\left(v_{k}\right)$ and $W \equiv h(V)$. Using the appropriate forms of (3.73) for $W_{k}$ and $w$, we get

(3.81) $\int_{\Omega} z_{, l}^{i}\left[C_{i j}^{l m}\left[x, v_{k}\right] w_{k, m}^{j}-C_{i j}^{l m}[x, v] w_{, m}^{j}\right] d x$

$$
\begin{aligned}
& +\int_{\Omega} z_{, \ell}^{i}\left[B_{i}^{\ell}\left[x, v_{k}\right]-B^{\ell}[x, V]\right] d x \\
& =\int_{\Omega} z^{i}\left[F_{i}\left[x, w_{k}\right]-F_{i}[x, W]\right] d x
\end{aligned}
$$

for all $\mathrm{z}$ in $\mathrm{H}_{0}^{l}(\Omega)$. This implies

(3.82) $\int_{\Omega} z_{, l}^{i} C_{i j}^{\ell m}\left[x, v_{k}\right]\left[w_{k}^{j}-w^{j}\right], m d x$

$$
\begin{aligned}
& +\int_{\Omega} z_{, l}^{i}\left[C_{i j}^{l m}\left[x, v_{k}\right]-C_{i j}^{\ell m}[x, v]\right] w_{, m}^{j} d x \\
& +\int_{\Omega} z_{, l}\left[B_{i}^{l}\left[x, v_{k}\right]-B_{i}^{l}[x, v]\right] d x \\
& =\int_{\Omega} z^{i}\left[F_{i}\left[x, w_{k}\right]-F_{i}[x, w]\right] d x .
\end{aligned}
$$

In (3.82), we put $z=W_{k}-W$ and use Assumption 3.2 to get 
(3.83) $\lambda \int_{\Omega}\left|z_{x}\right|^{2} d x$

$$
\begin{aligned}
& \leq \int_{\Omega}\left|z_{x}\right|\left|C\left[x, v_{k}\right]-C[x, v]\right|\left|w_{x}\right| d x \\
& +\int_{\Omega}\left|z_{x}\right|\left|B\left[x, v_{k}\right]-B[x, v]\right| d x \\
& +\int_{\Omega} z^{i}\left[F_{i}\left[x, w_{k}\right]-F_{i}[x, w]\right] d x .
\end{aligned}
$$

We now apply Lemma 2.2 to the first two integrals on the right hand side of $(3.83)$ to obtain

$$
\begin{aligned}
\text { (3.84) } & \lambda \int_{\Omega}\left|\mathrm{z}_{\mathrm{x}}\right|^{2} \mathrm{dx} \leq 2 \varepsilon \int_{\Omega}\left|\mathrm{z}_{\mathrm{x}}\right|^{2} \mathrm{dx} \\
+ & (4 \varepsilon)^{-1} \sum_{i, j=1} \sum_{\ell, \mathrm{m}=1}^{N} \int_{\Omega}\left|\mathrm{C}_{i j}^{\ell m}\left[\mathrm{x}, \mathrm{v}_{\mathrm{k}}\right]-\mathrm{C}_{\mathrm{ij}}^{\ell \mathrm{m}}[\mathrm{x}, \mathrm{v}]\right|^{2}\left|\mathrm{w}_{\mathrm{x}}\right|^{2} \mathrm{dx} \\
+ & \left.\sum_{i=1}^{N} \sum_{\ell=1}^{n} \int_{\Omega}\left|\mathrm{B}_{i}^{\ell}\left[\mathrm{x}, \mathrm{v}_{\mathrm{k}}\right]-\mathrm{B}_{i}^{\ell}[\mathrm{x}, \mathrm{v}]\right|^{2} \mathrm{dx}\right] \\
+ & \int_{\Omega} \mathrm{z}^{i}\left[\mathrm{~F}_{i}\left[\mathrm{x}, \mathrm{w}_{\mathrm{k}}\right]-\mathrm{F}_{\mathrm{i}}[\mathrm{x}, \mathrm{w}]\right] \mathrm{dx} .
\end{aligned}
$$

With the use of Assumption 3.2, we have

(3.85) $\int_{\Omega} z^{i}\left[F_{i}\left[x, W_{k}\right]-F_{i}[x, W]\right] d x \leq \eta \int_{\Omega}|z|^{2} d x$.

Using (3.85) in $(3.84)$ and simplifying, we get 


$$
(\lambda-2 \varepsilon) \int_{\Omega}\left|\mathrm{z}_{\mathrm{x}}\right|^{2} \mathrm{dx} \leq n \int_{\Omega}|\mathrm{z}|^{2} \mathrm{dx}
$$

$$
\begin{aligned}
& +(4 \varepsilon)^{-1} \sum_{i, j=1}^{N} \sum_{\ell, m=1}^{n} \int_{\Omega}\left|c_{i j}^{\ell m}\left[x, v_{k}\right]-c_{i j}^{\ell m}[x, v]\right|^{2}\left|w_{x}\right|^{2} d x \\
& +(4 \varepsilon)^{-1} \sum_{i=1}^{N} \sum_{\ell=1}^{n} \int_{\Omega}\left|B_{i}^{\ell}\left[x, v_{k}\right]-B_{i}^{\ell}[x, v]\right|^{2} d x .
\end{aligned}
$$

Let $\varepsilon=\min \left\{\left(\lambda-b^{2} n\right) / 4, \lambda / 4\right\}$ in $(3.86)$. If $n \leq 0$, then (3.86) implies

(3.87) $\int_{\Omega}\left|z_{x}\right|^{2} d x \leq\left(2 / \lambda^{2}\right)[\Delta B+\Delta C]$

where $\Delta C$ and $\Delta B$ are the last two integrals on the right hand side of (3.86), respectively. If $n \geq 0$, then Lemma 2.2 implies

(3.88) $\eta \int_{\Omega}|z|^{2} \mathrm{dx} \leq \mathrm{b}^{2} \eta \int_{\Omega}|\mathrm{z} \mathbf{x}|^{2} \mathrm{dx}$.

Using $(3.88)$ in $(3.86)$ yields

(3.89) $\int_{\Omega}\left|\mathrm{z}_{\mathrm{x}}\right|^{2} \mathrm{dx} \leq\left[2 /\left(\lambda-\mathrm{b}^{2} \eta\right)^{2}\right][\Delta \mathrm{B}+\Delta \mathrm{C}]$.

Combining (3.87) and (3.89), we get

(3.90) $\sum_{i=1}^{N} \int_{\Omega}\left|\left[w_{k}^{i}-w^{i}\right] x\right|^{2} d x \equiv \int_{\Omega}\left|z_{x}\right|^{2} d x$

$$
\leq \max \left\{2 / \lambda^{2}, 2 /\left(\lambda-b^{2} \eta\right)^{2}\right\}[\Delta B+\Delta C]
$$

By the continuity of $B$ given in Assumption 3.2, it is clear that $\Delta B$ of $(3.90)$ converges to zero as $k \rightarrow \infty$. The proof that $\Delta C$ converges to zero is done in the proof of Lemma 3.1 beginning with inequality $(3.26)$ and is therefore omitted 
here. This completes the proof.

LEMMA 3.10. The mapping $\mathrm{h}: \mathrm{L}_{2}(\Omega) \rightarrow \mathrm{L}_{2}(\Omega)$ is compact whenever Assumption 3.2 is valid.

PROOF. Since $h(V) \in H_{0}^{l}(\Omega)$ whenever $V \in L_{2}(\Omega)$, we need only show that $h(A)$ is bounded in $H_{0}^{1}(\Omega)$ whenever $A$ is a bounded set in $\mathrm{L}_{2}(\Omega)$. Theorem 2.1 then implies the lemma. Thus, suppose $A$ is a bounded set in $L_{2}(\Omega)$, and $K$ is a constant such that $A$ is contained in the set $\left\{\mathrm{V} \in \mathrm{L}_{2}(\Omega)|| \mid \mathrm{V} \|_{2, \Omega} \leq \mathrm{K}\right\}$. Let $\mathrm{V} \in \mathrm{A}$. We use Assumption 3.2, Lemma 2.2, and (3.73) with $z=W=h(V)$ to obtain

(3.91) $\lambda \int_{\Omega}\left|\mathrm{w}_{\mathrm{x}}\right|^{2} \mathrm{dx} \leq \int_{\Omega}\left|\mathrm{w}_{\mathrm{x}}\right||\mathrm{B}[\mathrm{x}, \mathrm{V}]| \mathrm{dx}+\int_{\Omega} \mathrm{w}^{i} \mathrm{~F}_{i}[\mathrm{x}, \mathrm{W}] \mathrm{dx}$

$$
\begin{aligned}
& \leq \varepsilon \int_{\Omega}\left|\mathrm{W}_{\mathrm{x}}\right|^{2} \mathrm{dx}+(4 \varepsilon)^{-1} \int_{\Omega}|\mathrm{B}[\mathrm{x}, \mathrm{V}]|^{2} \mathrm{dx} \\
& \quad+n \int_{\Omega}|\mathrm{W}|^{2} \mathrm{dx} .
\end{aligned}
$$

We let $\varepsilon=\tilde{\varepsilon} \equiv \min \left\{\lambda / 2,\left(\lambda-b^{2} \eta\right) / 2\right\}$ in $(3.91)$. If $\eta \leq 0$, then (3.91) yields

(3.92) $\quad|| \mathrm{W}_{\mathrm{x}}\left\|_{2, \Omega}^{2} \leq(2 \lambda \tilde{\varepsilon})^{-1}|| \mathrm{B}[\cdot, \mathrm{V}(\cdot)]\right\|_{2, \Omega}^{2}$

$$
\leq\left[\varepsilon_{1} /(2 \lambda \tilde{\varepsilon})\right]|| V||_{2, \Omega}^{2}+\left(M_{B} \operatorname{mes} \Omega\right) /(2 \lambda \tilde{\varepsilon}) .
$$

Corollary 2.1 and (3.92) imply

(3.93) ||$W||_{0, \Omega}^{2} \leq\left(1+b^{2}\right)\left[\varepsilon_{1} /(2 \lambda \tilde{\varepsilon})\right] \mathrm{K}^{2}$

$$
+\left(M_{B} \operatorname{mes} \Omega\right) /(2 \lambda \tilde{\varepsilon}) \text {. }
$$


If $\eta \geq 0$, then Lemma 2.1 along with (3.91) gives

$$
\text { (3.94) } \begin{aligned}
& \lambda|| \mathrm{w}_{\mathrm{x}}\left\|_{2, \Omega}^{2} \leq\left(\tilde{\varepsilon}+\mathrm{b}^{2} \eta\right)|| \mathrm{w}_{\mathbf{x}}\right\|_{2, \Omega}^{2} \\
+ & (4 \tilde{\varepsilon})^{-1}\|B[\cdot, \mathrm{V}(\cdot)]\|_{2, \Omega}^{2} \\
= & {\left[\left(\lambda+b^{2} \eta\right) / 2\right]\left\|\mathrm{w}_{\mathbf{x}}\right\|_{2, \Omega}^{2}+(4 \tilde{\varepsilon})^{-1}\|B[\cdot, \mathrm{V}(\cdot)]\|_{2, \Omega}^{2} }
\end{aligned}
$$

We now have from (3.94) and Assumption 3.2

$$
\begin{aligned}
\|\left. w_{x}\right|_{2, \Omega} ^{2} \leq & {\left[2 \tilde{\varepsilon}\left(\lambda-b^{2} \eta\right)\right]^{-1}|| B[\cdot, V(\cdot)] \|_{2, \Omega}^{2} } \\
\leq & {\left[\varepsilon_{1} / 2 \tilde{\varepsilon}\left(\lambda-b^{2} \eta\right)\right]|| v||_{2, \Omega}^{2} } \\
& +\left(M_{B} \operatorname{mes} \Omega\right) / 2 \tilde{\varepsilon}\left(\lambda-b^{2} \eta\right) .
\end{aligned}
$$

Corollary 2.1 and the definition of A imply

$$
\begin{aligned}
|| w||_{0, \Omega}^{2} \leq & {\left[\varepsilon_{1} / 2 \tilde{\varepsilon}\left(\lambda-b^{2} \eta\right)\right] \mathrm{k}^{2} } \\
& +\left(M_{B} \text { mes } \Omega\right) /\left[2 \tilde{\varepsilon}\left(\lambda-b^{2} n\right)\right],
\end{aligned}
$$

and the proof is complete.

LEMMA 3.11. Suppose Assumption 3.2 is valid and

$$
\begin{aligned}
& A \equiv\left\{\left.\mathrm{V} \varepsilon \mathrm{L}_{2}(\Omega)|||\mathrm{V}|\right|_{2, \Omega \leq \hat{\mathrm{K}}\} \text { where }}\right. \\
& \hat{\mathrm{K}} \equiv\left\{\left(\mathrm{b}^{2} \mathrm{M}_{\mathrm{B}} \operatorname{mes} \Omega\right) /\left[\left(\min \left\{\lambda, \lambda-\mathrm{b}^{2} \eta\right\}\right)^{2}-\mathrm{b}^{2} \varepsilon_{1}\right]\right\}^{1 / 2} \text {. Then } \\
& \mathrm{h}(\mathrm{A}) \subset \mathrm{A} \text {. } \\
& \quad \text { PROOF. If } \eta \leq 0 \text {, then }(3.92) \text { along with Lemma } 2.1 \\
& \text { implies }(\mathrm{W}=\mathrm{h}(\mathrm{V}))
\end{aligned}
$$


(3.97) ||$h(V)\left\|_{2, \Omega}^{2} \leq b^{2}\right\| h(V){ }_{x} \|_{2, \Omega}^{2}$

$$
\begin{aligned}
& \leq\left[b^{2} / 2 \lambda \tilde{\varepsilon}\right]\left[\varepsilon_{1}|| V||_{2, \Omega}^{2}+M_{B} \text { mes } \Omega\right] \\
& \leq\left[b^{2} / 2 \lambda \tilde{\varepsilon}\right]\left[\varepsilon_{I} \hat{\mathrm{K}}^{2}+\mathrm{M}_{B} \operatorname{mes} \Omega\right] \leq \hat{\mathrm{K}}^{2}
\end{aligned}
$$

If $\eta>0$, then (3.95) along with Lemma 2.1 implies

$$
\begin{aligned}
& \|h(V)\|_{2, \Omega \leq}^{2} \leq b^{2} \|\left. h(V){ }_{x}\right|_{2, \Omega} ^{2} \\
& \leq\left[b^{2} /\left\{2 \tilde{\varepsilon}\left(\lambda-b^{2} \eta\right)\right\}\right]\left\{\left.\varepsilon_{1}|| v\right|_{2, \Omega} ^{2}+M_{B} \text { mes } \Omega\right\} \\
& \leq\left[b^{2} /\left\{2 \tilde{\varepsilon}\left(\lambda-b^{2} \eta\right)\right\}\right]\left\{\varepsilon_{1} \hat{\mathrm{k}}^{2}+M_{B} \operatorname{mes} \Omega\right\} \leq \hat{\mathrm{k}}^{2},
\end{aligned}
$$

and the proof is complete.

THEOREM 3.4. Suppose Assumption 3.2 is valid. Then there is at least one solution to (1.1) and (1.2).

PROOF. Lemma 3.9 and Lemma 3.10 show that the mapping $h: L_{2}(\Omega) \rightarrow L_{2}(\Omega)$ is a compact map. Lemma 3.11 proves the existence of a closed bounded convex set $A$ in $L_{2}(\Omega)$ such that $\mathrm{h}(\mathrm{A}) \subset \mathrm{A}$. Theorem 2.2 implies $\mathrm{h}$ has a fixed point in $\mathrm{I}_{2}(\Omega)$, and the proof is complete. 
We shall study uniqueness and stability of the weak solution of (1.1) and (1.2) under stronger assumptions on the auxiliary functions than those needed for existence. Strengthening of Assumption 3.1 and Assumption 3.2 is obviously needed as indicated by the following example:

$(4.1)$

$$
\frac{d}{d x}\left(\frac{d U}{d x}-r(1-2 x) U^{(r-1) / r}\right)=0, x \in(0,1)
$$

$$
U(0)=U(I)=0 \text {. }
$$

The auxiliary functions involved in (4.1) clearly satisfy Assumption 3.1 and Assumption 3.2, but the problem has two solutions, $U=0$ and $U(x)=\left(x-x^{2}\right)^{r}$. Here, $r$ is any fixed number greater than 1 .

The following requirement imposed upon the auxiliary functions is motivated by a similar assumption used by Ford [5] in studying a two-point boundary value problem.

ASSUMPTION 4.1. Suppose there exist constants $n$ and $\gamma$ such that whenever $U, V, p^{l}, \ldots, p^{n}, q^{l}, \ldots, q^{n} \in E_{N}$ and $\mathbf{x} \varepsilon \Omega$,

$$
\text { (4.2) } \begin{aligned}
& \left(p_{\ell}^{i}-q_{\ell}^{i}\right)\left(C_{i j}^{\ell m}[x, U] p_{m}^{j}-C_{i j}^{\ell m}[x, v] q_{m}^{j}+B_{i}^{\ell}[x, U]\right. \\
& \left.-B_{i}^{\ell}[x, v]\right)-\left(U^{i}-v^{i}\right)\left(F_{i}[x, U]-F_{i}[x, U]\right)
\end{aligned}
$$




$$
\geq \gamma|p-q|-\eta|u-v|
$$

where

$$
\text { (4.3) } \quad \gamma \geq 0, \gamma-b^{2} \eta>0 \text {, }
$$

and $b$ is from (2.7).

When $\mathrm{n}=\mathrm{N}=1$ the above assumption reduces to a special case of a condition given by Ford [5] .

THEOREM 4.1. Suppose Assumption 4.1 is valid. Then there is at most one solution to (1.1) and (1.2).

PROOF. Suppose $U$ and $V$ are solutions of (1.1) and (1.2). This implies that U satisfies (1.12), and $V$ satisfies a similar equation. Taking the difference between the two expressions yields

$$
\text { (4.4) } \begin{aligned}
& \int_{\Omega} w_{, \ell}^{i}\left[C_{i j}^{\ell m}[x, U] U_{, m}^{j}-C_{i j}^{\ell m}[x, v] v_{, m}^{j}+B_{i}^{\ell}[x, U]\right. \\
& \left.+B_{i}^{l}[x, v]\right] d x+\int_{\Omega} w^{i}\left[F_{i}[x, U]-F_{i}[x, v]\right] d x=0
\end{aligned}
$$

for all $\mathrm{W}$ in $\mathrm{H}_{0}^{\mathrm{I}}(\Omega)$. Let $\mathrm{W}=\mathrm{U}-\mathrm{V}$ in (4.4), and use Assumption 4.1 to get

(4.5) $\lambda \int_{\Omega}\left|\mathrm{w}_{\mathbf{x}}\right|^{2} \mathrm{dx}-\eta \int_{\Omega}|\mathrm{w}|^{2} \mathrm{dx} \leq 0$.

If $\eta<0$, then (4.3) and (4.5) imply

(4.6) $-\eta \int_{\Omega}|w|^{2} d x \leq 0$,

and this implies 
(4.7) $\quad \int_{\Omega}|\mathrm{w}|^{2} \mathrm{dx} \leq 0$.

This yields $w=0$ a.e. If $n>0$, then (4.5) gives

$$
\gamma \int_{\Omega}\left|w_{x}\right|^{2} d x \leq n \int_{\Omega}|w|^{2} d x .
$$

Lemma 2.1 with $p=2$ yields the estimate

$$
\eta \int_{\Omega}|w|^{2} d x \leq n b^{2} \int_{\Omega}\left|w_{x}\right|^{2} d x
$$

Combining (4.8) and (4.9) produces

$(4.10)$

$$
\left(r-b^{2} n\right) \int_{\Omega}\left|w_{x}\right|^{2} d x \leq 0 .
$$

Inequalities (4.3) and (4.10) imply

$$
\text { (4.11) } \quad \int_{\Omega}\left|w_{x}\right|^{2} d x \leq 0
$$

which yields $W=0$ a.e. and the proof is complete.

By requiring $\gamma>0$ in Assumption 4.1, we can obtain a stability condition for the problem (1.1) and (1.2).

THEOREM 4.2. Suppose Assumption 4.1 is valid with $\gamma>0$, and $U$ is a solution of (1.1) and (1.2) with $\left|U_{x}\right| \varepsilon L_{2 p}(\Omega)$, $\mathrm{p} \geq 1$. If $\tilde{U}$ is a solution (1.1) and (1.2) with auxiliary functions $\tilde{C}, \tilde{B}$, and $\tilde{F}$ satisfying Assumption $4.1(\gamma>0)$ with all constants the same as for C, B, and F, then there exists a positive constant $\mathrm{K}_{\mathrm{o}}$ depending only on known constants such that 


$$
\text { (4.12) } \begin{aligned}
& \|\mathrm{U}-\tilde{\mathrm{U}}\|_{0, \Omega} \\
& \leq \mathrm{K}_{\mathrm{O}}\left[|| \mathrm{U}_{\mathrm{X}}\left\|_{2 \mathrm{p}, \Omega}|| \mathrm{C}[\cdot, \mathrm{U}(\cdot)]-\tilde{\mathrm{C}}[\cdot, \mathrm{U}(\cdot)]\right\|_{2 \mathrm{p}^{\prime}, \Omega}\right. \\
& \quad+\|\mathrm{B}[\cdot, \mathrm{U}(\cdot)]-\tilde{\mathrm{B}}[\cdot, \mathrm{U}(\cdot)]\|_{2, \Omega} \\
& \left.\quad+\|\mathrm{F}[\cdot, \mathrm{U}(\cdot)]-\tilde{\mathrm{F}}[\cdot, \mathrm{U}(\cdot)]\|_{2, \Omega}\right]
\end{aligned}
$$

where $(1 / p)+\left(1 / p^{\prime}\right)=1$

PROOF. Using (1.12) and the corresponding form of (1.12) for $\tilde{U}$, we take the difference between the two equations to obtain

(4.13) $\int_{\Omega} z_{, l}^{i}\left[C_{i j}^{\ell m}[x, U] U_{, m}^{j}-\tilde{C}_{i j}^{\ell m}[x, \tilde{U}] \tilde{U}_{, m}^{j}+B_{i}^{\ell}[x, U]\right.$

$$
\left.-\tilde{B}_{i}^{\ell}[x, \tilde{U}]\right] d x-\int_{\Omega} z^{i}\left[F_{i}[x, U]-\tilde{F}_{i}[x, \tilde{U}] d x=0 .\right.
$$

By adding and subtracting $\tilde{C}_{i j}^{l m}[x, U] U_{,}^{j}, \tilde{B}_{i}^{\ell}[x, U]$, and $\tilde{F}_{i}[x, U]$ to the integrands and collecting terms, we get

$$
\text { (4.14) } \begin{aligned}
& \int_{\Omega} z_{, l}^{i}\left[\tilde{C}_{i j}^{l m}[x, U] U_{, m}^{j}-\tilde{C}_{i j}^{l m}[x, \tilde{U}] \tilde{U}_{, m}^{j}+\tilde{B}_{i}^{l}[x, U]\right. \\
& \left.-\tilde{B}_{i}^{\ell}[x, \tilde{U}]\right] d x-\int_{\Omega} z^{i}\left[\tilde{F}_{i}[x, U]-\tilde{F}_{i}[x, \tilde{U}]\right] d x \\
= & \int_{\Omega} z_{, l}^{i}\left[\tilde{C}_{i j}^{l m}[x, U] U_{, m}^{j}-C_{i j}^{l m}[x, U] U_{, m}^{j}\right] d x \\
& +\int_{\Omega} z_{, l}^{i}\left[\tilde{B}_{i}^{\ell}[x, U]-B_{i}^{l}[x, U]\right] d x
\end{aligned}
$$




$$
-\int_{\Omega} z^{i}\left[\tilde{F}_{i}[x, U]-F_{i}[x, U]\right] d x
$$

for all $\mathrm{Z}$ in $\mathrm{H}_{0}^{l}(\Omega)$. Now let $\mathrm{Z}=\mathrm{U}-\tilde{\mathrm{U}}$, and apply Assumption 4.1 to the left hand side of (4.14) to obtain

$$
\text { (4.15) } \begin{aligned}
& \gamma \int_{\Omega}\left|z_{x}\right|^{2} d x-n \int_{\Omega}|z|^{2} d x \\
& \leq \int_{\Omega} z_{, l}^{i}\left[\tilde{C}_{i j}^{l m}[x, U]-c_{i j}^{l m}[x, U]\right] u_{, m}^{j} d x \\
&+\int_{\Omega} z_{, l}^{i}\left[\tilde{B}_{i}^{l}[x, U]-B_{i}^{l}[x, U]\right] d x \\
&-\int_{\Omega} z^{i}\left[\tilde{F}_{i}[x, U]-F_{i}[x, U]\right] d x .
\end{aligned}
$$

We now use Lemma 2.2 to obtain the following estimates for the integrals on the right hand side of (4.15). For $\varepsilon>0$, we have

(4.16) $\int_{\Omega} z_{, \ell}^{i}\left[\tilde{C}_{i j}^{\ell m}[x, U]-c_{i j}^{\ell m}[x, U]\right] U_{, m}^{j} d x$

$$
\leq \int_{\Omega}\left|z_{x}\right|^{2} d x+(4 \varepsilon)^{-1} \int_{\Omega}|\tilde{c}[x, U]-c[x, U]|^{2}\left|U_{x}\right|^{2} d x .
$$

(4.17) $\int_{\Omega} \mathrm{z}_{, l}^{i}\left[\tilde{B}_{i}^{\ell}[\mathrm{x}, \mathrm{U}]-\mathrm{B}_{i}^{\ell}[\mathrm{x}, \mathrm{U}]\right] \mathrm{dx} \leq \varepsilon \int_{\Omega}\left|\mathrm{z}_{\mathrm{x}}\right|^{2} \mathrm{dx}$

$$
+(4 \varepsilon)^{-1} \int_{\Omega}|\tilde{B}[\mathrm{x}, \mathrm{U}]-\mathrm{B}[\mathrm{x}, \mathrm{U}]|^{2} \mathrm{dx}
$$

(4.18) $\int_{\Omega} z^{i}\left[\tilde{F}_{i}[x, U]-F_{i}[x, U]\right] d x \leq \varepsilon \int_{\Omega}|z|^{2} d x$ 


$$
\begin{aligned}
& +(4 \varepsilon)^{-1} \int_{\Omega}|\tilde{F}[\mathrm{x}, \mathrm{U}]-\mathrm{F}[\mathrm{x}, \mathrm{U}]|^{2} \mathrm{dx} \\
& \leq \varepsilon \mathrm{b}^{2} \int_{\Omega}\left|\mathrm{z}_{\mathrm{x}}\right|^{2} \mathrm{dx}+(4 \varepsilon)^{-1} \int_{\Omega}|\tilde{F}[\mathrm{x}, \mathrm{U}]-\mathrm{F}[\mathrm{x}, \mathrm{U}]|^{2} \mathrm{dx}
\end{aligned}
$$

where $b$ is from (2.7). Combining (4.15) through (4.18)

yields

$$
\begin{aligned}
& \text { (4.19) } \gamma \int_{\Omega}\left|z_{x}\right|^{2} d x-\eta \int_{\Omega}|z|^{2} d x \leq \varepsilon\left(2+b^{2}\right) \int_{\Omega}\left|z_{x}\right|^{2} d x \\
& +(4 \varepsilon)^{-1}\left[\int_{\Omega}|\tilde{c}[x, U]-c[x, U]|^{2}\left|U_{x}\right|^{2} d x\right. \\
& +\int_{\Omega}|\tilde{B}[\mathrm{x}, \mathrm{U}]-\mathrm{B}[\mathrm{x}, \mathrm{U}]|^{2} \mathrm{dx} \\
& \left.+\int_{\Omega}|\tilde{F}[x, U]-F[x, U]|^{2} d x\right]
\end{aligned}
$$

We also have from Hölder's inequality

$$
\text { (4.20) } \begin{aligned}
\int_{\Omega}|\tilde{C}[\mathrm{x}, \mathrm{U}]-\mathrm{C}[\mathrm{x}, \mathrm{U}]|^{2}\left|\mathrm{U}_{\mathrm{x}}\right|^{2} \mathrm{dx} \\
\leq|| \mathrm{u}_{\mathrm{x}}\left\|_{2 \mathrm{p}, \Omega}^{2}|| \tilde{\mathrm{C}}[\cdot, \mathrm{U}(\cdot)]-\mathrm{C}[\cdot, \mathrm{U}(\cdot)]\right\|_{2 \mathrm{p}^{\prime}, \Omega}^{2}
\end{aligned}
$$

If $n \leq 0$, then

(4.21) $\gamma \int_{\Omega}\left|z_{x}\right|^{2} d x-\eta \int_{\Omega}|z|^{2} d x \geq \gamma \int_{\Omega}\left|z_{x}\right|^{2} d x$.

On the other hand, if $n>0$, Lemma 2.1 with $p=2$ implies

(4.22) $\gamma \int_{\Omega}|z|^{2} d x-\eta \int_{\Omega}|z|^{2} \mathrm{dx} \geq\left(\gamma-\mathrm{b}^{2} \eta\right) \int_{\Omega}\left|z_{\mathrm{x}}\right|^{2} \mathrm{dx}$.

We define $\delta_{0} \equiv \min \left\{\gamma, \gamma-b^{2} \eta\right\}$. Then (4.21) and (4.22) 
imply

(4.23) $\delta_{\circ} \int_{\Omega}\left|z_{x}\right|^{2} d x \leq \gamma \int_{\Omega}\left|z_{x}\right|^{2} d x-\eta \int_{\Omega}|z|^{2} d x$.

substituting (4.20) and (4.23) into (4.19) and collecting like terms, we get

$(4.24)$

$$
\begin{aligned}
& {\left[\delta_{0}-\varepsilon\left(2+\mathrm{b}^{2}\right)\right] \int_{\Omega}\left|\mathrm{z}_{\mathrm{x}}\right|^{2} \mathrm{dx}} \\
& \leq(4 \varepsilon)^{-1}\left[|| \mathrm{U}_{\mathrm{x}}||_{2 \mathrm{p}, \Omega}^{2}|| \mathrm{C}[\cdot, \mathrm{U}(\cdot)]-\tilde{\mathrm{C}}[\cdot, \mathrm{U}(\cdot)]||_{2 \mathrm{p}^{\prime}, \Omega}^{2}\right. \\
& \quad+|| \mathrm{B}[\cdot, \mathrm{U}(\cdot)]-\tilde{\mathrm{B}}[\cdot, \mathrm{U}(\cdot)]||_{2, \Omega}^{2} \\
& \left.+|| \mathrm{F}[\cdot, \mathrm{U}(\cdot)]-\left.\tilde{\mathrm{F}}[\cdot, \mathrm{U}(\cdot)]\right|_{2, \Omega} ^{2}\right] .
\end{aligned}
$$

Let $\varepsilon=\delta_{0}\left[2\left(2+b^{2}\right)\right]^{-1}$ in $(4.24)$ and use corollary 2.1 to obtain (4.12) with $\mathrm{K}_{0}=\left\{\left[\left(1+\mathrm{b}^{2}\right)\left(2+\mathrm{b}^{2}\right)\right]^{1 / 2}\right\} / \delta_{0}$. This completes the proof.

A slightly stronger stability result is now given in which no extra smoothness condition on any solution is required.

THEOREM 4.3. Suppose the sequences $\left\{C_{i j k}^{l m}\right\}_{k=1}^{\infty},\left\{B_{i k}^{l}\right\}_{k=1}^{\infty}$, and $\left\{F_{i k}\right\}_{k=1}^{\infty}(1 \leq i, j \leq N$ and $l \leq \ell, m \leq n)$ of functions from $\Omega \times E_{N}$ into $E_{1}$ satisfy the conditions of Assumption 4.1 and $\left\{C_{i j k}^{l m}\right\}_{k=1}^{\infty}$ satisfy inequality (3.2) with the constants the same as those for $C_{i j}^{l m}, B_{i}^{l}$, and $F_{i}$. If $C_{i j k}^{l m}[\cdot, V(\cdot)]$, $\mathrm{B}_{i \mathrm{k}}^{\ell}[\cdot, \mathrm{V}(\cdot)]$, and $\mathrm{F}_{i \mathrm{k}}[\cdot, \mathrm{V}(\cdot)]$ converge to $\mathrm{C}_{\mathrm{ij}}^{\ell \mathrm{m}}[\cdot, \mathrm{V}(\cdot)]$, $B_{i}^{l}[\cdot, V(\cdot)]$, and $F_{i}[\cdot, V(\cdot)]$, respectively, in $L_{2}(\Omega)$ for each 
$\mathrm{V}$ in $\mathrm{L}_{2}(\Omega)$ as $\mathrm{k}$ approaches $\infty$, then the sequence of weak solutions $\left\{U_{k}\right\}_{k=1}^{\infty}$ converges to $U$ in $H_{0}^{l}(\Omega)$.

PROOF. The analysis is identical to that in the proof of Theorem 4.2 through inequality (4.19) with $\tilde{C}_{i j}^{l m}, \tilde{B}_{i}^{l}, \tilde{F}_{i}$, and $\tilde{U}$ replaced by $C_{i j k}^{l m}, B_{i k}^{l}, F_{i k}$, and $U_{k}$, respectively. We then apply inequalities (4.19) and (4.23) to get

$(4.25)$

$$
\begin{aligned}
& || \mathrm{U}-\mathrm{U}_{\mathrm{k}} \|_{0, \Omega} \\
& \leq \mathrm{K}_{\mathrm{O}}\left[\|\left.\left|\mathrm{C}[\cdot, \mathrm{U}(\cdot)]-\mathrm{C}_{\mathrm{k}}[\cdot, \mathrm{U}(\cdot)]\right|\left|\mathrm{U}_{\mathrm{x}}\right|\right|_{2, \Omega}\right. \\
& \quad+\mid \mathrm{B}[\cdot, \mathrm{U}(\cdot)]-\mathrm{B}_{\mathrm{k}}[\cdot, \mathrm{U}(\cdot)] \|_{2, \Omega} \\
& \left.\quad+|| \mathrm{F}[\cdot, \mathrm{U}(\cdot)]-\mathrm{F}_{\mathrm{k}}[\cdot, \mathrm{U}(\cdot)] \|_{2, \Omega}\right]
\end{aligned}
$$

where $k_{0}$ is from Theorem 4.2. From (4.25) and the hypothesis of the theorem, it suffices to show

$$
\text { (4.26) }\left.\lim _{\mathrm{k} \rightarrow \infty}||\left|\mathrm{C}_{\mathrm{k}}[\cdot, \mathrm{U}(\cdot)]-\mathrm{C}[\cdot, \mathrm{U}(\cdot)]\right|\left|\mathrm{U}_{\mathrm{x}}\right|\right|_{2, \Omega}=0
$$

since the last two terms in $(4.25)$ clearly converge to zero as $k$ approaches $\infty$. To prove $(4.26)$, we let $\delta>0$ be given. We choose a sequence $\left\{\mathrm{w}_{j}\right\}_{j=1}^{\infty} \subset \mathrm{C}_{0}^{\infty}(\Omega)$ such that $\lim _{j+\infty}|| w_{j}-\left.U\right|_{0, \Omega}=0$. Suppose $A_{j}$ is a constant such that $\left.|| w_{j_{x}}\right|_{\infty, \Omega} \leq A_{j}, j=1,2, \ldots$ Choose a positive integer $J(\delta)$ depending only on $\delta$ such that ||$W_{J}(\delta)_{\mathbf{X}}-U_{\mathbf{X}} \|_{2, \Omega}<\delta / 4 M_{C}$ where $M_{C}$ is the constant from (3.2). We now choose $K(J(\delta)$ ) 
depending only on $J(\delta)$ such that $k \geq K(J(\delta))$ implies

$$
(4.27)|| C_{k}[\cdot, U(\cdot)]-C[\cdot, U(\cdot)] \|_{2, \Omega}<\delta /\left(2 A_{J}(\delta)\right) .
$$

We now have the following sequence of inequalities:

$$
\text { (4.28) } \begin{aligned}
& \|\left.\left|\mathrm{C}_{\mathrm{k}}[\cdot, \mathrm{U}(\cdot)]-\mathrm{C}[\cdot, \mathrm{U}(\cdot)]\right|\left|\mathrm{U}_{\mathrm{x}}\right|\right|_{2, \Omega} \\
\leq & \left.||\left|\mathrm{C}_{\mathrm{k}}[\cdot, \mathrm{U}(\cdot)]-\mathrm{C}[\cdot, \mathrm{U}(\cdot)]\right|\left|\mathrm{U}_{\mathrm{x}}-\mathrm{W}_{\mathrm{J}(\delta)}\right|\right|_{2, \Omega} \\
& +\left.||\left|\mathrm{C}_{\mathrm{k}}[\cdot, \mathrm{U}(\cdot)]-\mathrm{C}[\cdot, \mathrm{U}(\cdot)]\right|\left|\mathrm{W}_{\mathrm{J}(\delta)_{\mathrm{x}}}\right|\right|_{2, \Omega} \\
\leq & 2 \mathrm{M}_{\mathrm{C}}|| \mathrm{U}_{\mathrm{x}}-\mathrm{W}_{\mathrm{J}(\delta)_{\mathrm{x}}||_{2, \Omega}} \\
& +\mathrm{A}_{\mathrm{J}(\delta)}|| \mathrm{C}_{\mathrm{k}}[\cdot, \mathrm{U}(\cdot)]-\left.\mathrm{C}[\cdot, \mathrm{U}(\cdot)]\right|_{2, \Omega} \\
< & (\delta / 2)+(\delta / 2)=\delta
\end{aligned}
$$

whenever $k \geq K(J(\delta))$, and thus, (4.26) is proven. This completes the proof. 


\section{CHAPTER V}

\section{BOUNDEDNESS OF WEAK SOLUTIONS}

We now show that under more stringent restrictions on the auxiliary functions than those used for existence all weak solutions of (1.1) and (1.2) are bounded (a.e.) by a constant depending only on the constants of Assumption 3.1 and other known constants.

ASSUMPTION 5.1. Suppose Assumption 3.1 is satisfied except for inequalities (3.3), (3.4), and (3.5). In place of these, we suppose there exist nonnegative constants $M$ and $\alpha$ such that

$$
|F[x, U]|+|B[x, U]| \leq M\left[1+|U|^{\alpha}\right]
$$

for all $x \in \Omega$ and $U \varepsilon E_{N}$ where $\alpha<\min \{1,2 /(n-2)\}$ for $\mathrm{n}=2,3,4$, or 5 and $\alpha=1 / 2$ for $\mathrm{n} \geq 6$. Furthermore, suppose

$$
C_{i j}^{\ell m}[x, U]=0 \text { for } i \neq j, x \in \Omega, U \in E_{N} \text {. }
$$

Before proving boundedness of the weak solutions in the $L_{\infty}(\Omega)$ norm, we consider some preliminary lemmas.

LEMMA 5.1. Suppose Assumption 3.1 is valid, and $U$ is a solution of (1.1) and (1.2). Then there exists a constant Mo depending only on known constants such that

$$
\left.|| \mathrm{U}_{\mathrm{x}}\right|_{2, \Omega} \leq \mathrm{M}_{\mathrm{O}} \text {. }
$$


PROOF. In (1.12) put $\mathrm{z}=\mathrm{U}$ and use Assumption 3.1 along with Lemma 2.2 to get

$$
\begin{aligned}
\lambda \int_{\Omega}\left|\mathrm{U}_{\mathbf{x}}\right|^{2} \mathrm{dx} & \leq \int_{\Omega}\left|\mathrm{U}_{\mathrm{x}}\right||\mathrm{B}| \mathrm{d} \mathrm{x}+\int_{\Omega}|\mathrm{U}||\mathrm{F}| \mathrm{d} \mathbf{x} \\
& \leq \varepsilon \int_{\Omega}\left|\mathrm{U}_{\mathrm{x}}\right|^{2} \mathrm{dx}+(4 \varepsilon)^{-1} \int_{\Omega}|\mathrm{B}|^{2} \mathrm{dx} \\
& +\varepsilon \int_{\Omega}|\mathrm{U}|^{2} \mathrm{dx}+(4 \varepsilon)^{-1} \int_{\Omega}|\mathrm{F}|^{2} \mathrm{dx} .
\end{aligned}
$$

Assumption 3.1 and Lemma 2.1 imply

$(5.5) \quad(4 \varepsilon)^{-1} \int_{\Omega}\left[|B|^{2}+|F|^{2}\right] \mathrm{dx}$

$$
\begin{aligned}
& \leq\left(\varepsilon_{0}+\varepsilon_{1}\right) / 4 \varepsilon \int_{\Omega}|U|^{2} \mathrm{dx} \\
& +\left[\left(M_{F}+M_{B}\right) \text { mes } \Omega\right] / 4 \varepsilon .
\end{aligned}
$$

Inequalities (5.4) and (5.5) combined with Lemma 2.1 yield

$$
\text { (5.6) } \begin{aligned}
\lambda & \int_{\Omega}\left|\mathrm{U}_{\mathbf{x}}\right|^{2} \mathrm{dx} \\
& \leq\left[\varepsilon\left(1+\mathrm{b}^{2}\right)+\left[\left(\varepsilon_{0}+\varepsilon_{1}\right) \mathrm{b}^{2} / 4 \varepsilon\right]\right] \int_{\Omega}\left|\mathrm{U}_{\mathbf{x}}\right|^{2} \mathrm{dx} \\
& +\left[\left(\mathrm{M}_{\mathrm{F}}+\mathrm{M}_{\mathrm{B}}\right) \text { mes } \Omega\right] / 4 \varepsilon .
\end{aligned}
$$

In (5.6) we let $\varepsilon=\lambda\left[2\left(1+b^{2}\right)\right]^{-1}$ and use (3.3) to get

(5.7) $\lambda \int_{\Omega}\left|\mathrm{U}_{\mathbf{x}}\right|^{2} \mathrm{dx} \leq(\lambda / 2)\left[\left(1+2 \mathrm{~b}^{2}\right) /\left(1+\mathrm{b}^{2}\right)\right] \int_{\Omega}\left|\mathrm{U}_{\mathbf{x}}\right|^{2} \mathrm{dx}$

$$
+\left[\left(M_{F}+M_{B}\right)\left(1+b^{2}\right) / 2 \lambda\right] \text { mes } \Omega \text {. }
$$


Simplification of (5.7) yields (5.3), and the proof is complete.

LEMMA 5.2. Suppose Assumption 5.1 is valid with $n \geq 6$. Then there exists a constant $\overline{\bar{M}}(s)$ depending only on known constants and the positive integer s such that

$$
\int_{\Omega}|\mathrm{U}|^{4 \mathrm{~s}} \mathrm{dx} \leq \overline{\overline{\mathrm{M}}}(\mathrm{s})
$$

for each $s=1,2,3, \ldots$

PROOF. Let $k$ be a fixed integer such that $1 \leq k \leq N$. Let $\mathrm{V}=\left(0, \ldots, 0, \mathrm{U}^{\mathrm{k}}, 0, \ldots, \mathrm{Q}\right)$ where the $\mathrm{U}^{\mathrm{k}}$ appears in the $k$-th position of the vector $v$. The proof is by induction on $s$. Thus, suppose $s=1$. In (1.12), let $\mathrm{Z}(\mathrm{x})=\mathrm{V}(\mathrm{x}) \Phi(\mathrm{x})$ where $\Phi(\mathrm{x}) \equiv \min \left\{|\mathrm{V}(\mathrm{x})|^{2}, \mathrm{R}\right\}$ and $\mathrm{R}>0$. Clearly, $\mathrm{Z}$ is an element of $\mathrm{H}_{0}^{1}(\Omega)$. With this value of $\mathrm{Z}$, we get

$$
\begin{aligned}
& \int_{\Omega} \Phi v_{, l}^{i} c_{i j}^{\ell m} U_{, m}^{j} d x+\int_{\Omega} \Phi, l c_{i j}^{\ell m} v^{i} U_{, m}^{j} d x \\
& =-\int_{\Omega} \mathrm{v}_{, l}^{i} \Phi \mathrm{B}_{i}^{\ell} \mathrm{dx}-\int_{\Omega} \mathrm{v}^{i} \Phi, \ell \mathrm{B}_{i}^{\ell} \mathrm{dx}+\int_{\Omega} \mathrm{v}^{i} \Phi \mathrm{F}_{i} \mathrm{dx}
\end{aligned}
$$

Assumption 5.1 and the definition of $\Phi$ applied to (5.9) give

$$
\begin{aligned}
(5.10) & \lambda \int_{\Omega} \Phi\left|\mathrm{v}_{\mathrm{x}}\right|^{2} \mathrm{~d} \mathrm{x}+(\lambda / 2) \int_{\Omega}\left|\Phi_{\mathrm{x}}\right|^{2} \mathrm{dx} \\
\leq & \int_{\Omega}\left|\mathrm{v}_{\mathrm{x}}\right||\Phi|\left|\mathrm{B}_{\mathrm{k}}\right| \mathrm{d} \mathrm{x}+\int_{\Omega}|\mathrm{V}|\left|\Phi_{\mathrm{x}}\right|\left|\mathrm{B}_{\mathrm{k}}\right| \mathrm{d} \mathbf{x} \\
& +\int_{\Omega}|\mathrm{V}||\Phi|\left|\mathrm{F}_{\mathrm{k}}\right| \mathrm{dx}
\end{aligned}
$$


where $\left|B_{k}\right| \equiv\left[\sum_{\ell=1}^{n}\left|B_{k}^{\ell}\right|^{2}\right]^{1 / 2}$. We now use Assumption 5.1 and Lemma 2.2 to estimate the integrals on the right hand side of $(5.10)$.

(5.11) $\int_{\Omega}\left|\mathrm{v}_{\mathbf{x}}\right| \Phi\left|\mathrm{B}_{\mathrm{k}}\right| \mathrm{dx} \leq \mathrm{M} \int_{\Omega}\left|\mathrm{v}_{\mathbf{x}}\right| \Phi \mathrm{dx}$

$$
\begin{aligned}
& +\mathrm{M} \int_{\Omega}\left|\mathrm{V}_{\mathrm{X}}\right| \Phi|\mathrm{U}|^{1 / 2} \mathrm{dx} \\
& \leq 2 \varepsilon \int_{\Omega}\left|\mathrm{V}_{\mathrm{X}}\right|^{2} \Phi \mathrm{dx}+\left(\mathrm{M}^{2} / 4 \varepsilon\right) \int_{\Omega} \Phi \mathrm{dx} \\
& +\left(\mathrm{M}^{2} / 4 \varepsilon\right) \int_{\Omega} \Phi|\mathrm{U}| \mathrm{dx} \\
& \leq 2 \varepsilon \int_{\Omega}\left|\mathrm{V}_{\mathrm{X}}\right|^{2} \Phi \mathrm{dx}+2 \varepsilon \int_{\Omega}|\Phi|^{2} \mathrm{dx} \\
& +\left[\mathrm{M}^{4} /\left(64 \varepsilon^{3}\right)\right]\left[\text { mes } \Omega+\int_{\Omega}|\mathrm{U}|^{2} \mathrm{dx}\right]
\end{aligned}
$$

where $b$ is from (2.7).

(5.12) $\int_{\Omega}|\mathrm{V}|\left|\Phi_{\mathrm{x}}\right|\left|\mathrm{B}_{\mathrm{k}}\right| \mathrm{dx} \leq \mathrm{M} \int_{\Omega}|\mathrm{V}|\left|\Phi_{\mathrm{x}}\right| \mathrm{dx}$

$$
\begin{aligned}
& +M \int_{\Omega}|V|\left|\Phi_{x}\right||U|^{1 / 2} d x \\
& =M \int_{\Omega}(\Phi)^{1 / 2}\left|\Phi_{\mathbf{x}}\right| \mathrm{dx}+M \int_{\Omega}(\Phi)^{1 / 2}\left|\Phi_{\mathbf{X}}\right||\mathrm{U}|^{1 / 2} \mathrm{dx} \\
& \leq 2 \varepsilon \int_{\Omega}\left|\Phi_{\mathrm{X}}\right|^{2} \mathrm{dx}+\left(\mathrm{M}^{2} / 4 \varepsilon\right)\left[\int_{\Omega} \Phi \mathrm{dx}\right. \\
& \left.+\int_{\Omega} \Phi|\mathrm{U}| \mathrm{dx}\right]
\end{aligned}
$$




$$
\begin{aligned}
& \leq \quad 2 \varepsilon \int_{\Omega}\left|\Phi_{\mathrm{x}}\right|^{2} \mathrm{dx}+2 \varepsilon \int_{\Omega}|\Phi|^{2} \mathrm{dx} \\
& +\left(\mathrm{M}^{4} / 64 \varepsilon^{3}\right)\left[\text { mes } \Omega+\int_{\Omega}|\mathrm{U}|^{2} \mathrm{dx}\right] \\
& \leq 2 \varepsilon\left(1+\mathrm{b}^{2}\right) \int_{\Omega}\left|\Phi_{\mathrm{x}}\right|^{2} \mathrm{dx} \\
& +\left(\mathrm{M}^{4} / 64 \varepsilon^{3}\right)\left[\text { mes } \Omega+\int_{\Omega}|\mathrm{U}|^{2} \mathrm{dx}\right] .
\end{aligned}
$$

We now use the inequality (no summation on $\mathrm{m}$ )

$$
\left|\left[\left(|\mathrm{V}|^{2} \Phi\right)^{1 / 2}\right]_{, m}\right|^{2} \leq 4 \Phi\left|v_{, m}\right|^{2}, 1 \leq m \leq n,
$$

from [8, p. 392] in estimating the last term in (5.10). Thus, we obtain

(5.14) $\int_{\Omega}|\mathrm{V}| \Phi\left|\mathrm{F}_{\mathrm{k}}\right| \mathrm{dx} \leq \mathrm{M} \int_{\Omega}|\mathrm{V}| \Phi \mathrm{dx}$

$$
\begin{aligned}
& +\mathrm{M} \int_{\Omega}|\mathrm{V}| \Phi|\mathrm{U}|^{1 / 2} \mathrm{dx} \\
& \leq 2 \varepsilon \int_{\Omega}|\mathrm{V}|^{2} \Phi \mathrm{d} \mathbf{x}+\left(\mathrm{M}^{2} / 4 \varepsilon\right)\left[\int_{\Omega} \Phi \mathrm{d} \mathrm{x}+\int_{\Omega} \Phi|\mathrm{U}| \mathrm{dx}\right] \\
& \leq \quad \varepsilon \int_{\Omega}\left[\left(|\mathrm{V}|^{2} \Phi\right)^{1 / 2}\right]^{2} \mathrm{dx}+2 \varepsilon \int_{\Omega}|\Phi|^{2} \mathrm{dx} \\
& +\left(\mathrm{M}^{4} / 64 \varepsilon^{3}\right)\left[\text { mes } \Omega+\int_{\Omega}|\mathrm{U}|^{2} \mathrm{dx}\right] \\
& \leq 2 \varepsilon \mathrm{b}^{2} \int_{\Omega}\left|\left[\left(|\mathrm{V}|^{2} \Phi\right)^{1 / 2}\right]\right|^{2} \mathrm{dx}+2 \varepsilon \mathrm{b}_{\Omega}|\Phi \mathrm{x}|^{2} \mathrm{dx} \\
& +\left(\mathrm{M}^{4} / 64 \varepsilon^{3}\right)\left[\text { mes } \Omega+\int_{\Omega}|\mathrm{U}|^{2} \mathrm{dx}\right]
\end{aligned}
$$




$$
\begin{aligned}
\leq & 8 \varepsilon b^{2} \int_{\Omega} \Phi\left|\mathrm{V}_{\mathbf{x}}\right|^{2} \mathrm{dx}+2 \varepsilon \mathrm{b}^{2} \int_{\Omega}\left|\Phi_{\mathrm{x}}\right|^{2} \mathrm{dx} \\
& +\left(\mathrm{M}^{4} / 64 \varepsilon^{3}\right)\left[\text { mes } \Omega+\int_{\Omega}|\mathrm{U}|^{2} \mathrm{dx}\right]
\end{aligned}
$$

where we have used (5.13) to get the last inequality. We now may combine $(5.10)$ through $(5.14)$ to obtain

$$
\text { (5.15) } \quad \begin{aligned}
& \lambda \int_{\Omega} \Phi\left|\mathrm{v}_{\mathrm{x}}\right|^{2} \mathrm{dx}+(\lambda / 2) \int_{\Omega}\left|\Phi_{\mathrm{x}}\right|^{2} \mathrm{dx} \\
& \leq 2 \varepsilon\left[1+4 \mathrm{~b}^{2}\right] \int_{\Omega} \Phi\left|\mathrm{v}_{\mathrm{x}}\right|^{2} \mathrm{dx} \\
&+2 \varepsilon\left[1+3 \mathrm{~b}^{2}\right] \int_{\Omega}\left|\Phi_{\mathrm{x}}\right|^{2} \mathrm{dx} \\
&\left.+\left(3 \mathrm{M}^{4} / 64 \varepsilon^{3}\right) \text { [mes } \Omega+\int_{\Omega}|\mathrm{U}|^{2} \mathrm{dx}\right] .
\end{aligned}
$$

Choose $\varepsilon=\lambda\left[8\left(1+4 b^{2}\right)\right]^{-1}$ in $(5.15)$ and collect like terms to get

$$
\begin{aligned}
\text { (5.16) }(3 \lambda / 4) \int_{\Omega} \Phi\left|\mathrm{v}_{\mathrm{x}}\right|^{2} \mathrm{dx}+(\lambda / 4) \int_{\Omega}\left|\Phi_{\mathrm{x}}\right|^{2} \mathrm{dx} \\
\leq \mathrm{K}_{\mathrm{O}}(\lambda, \operatorname{mes} \Omega, \mathrm{M}, \mathrm{b}, \mathrm{n})+\mathrm{K}_{1}(\lambda, \mathrm{M}) \int_{\Omega}|\mathrm{U}|^{2} \mathrm{dx} \\
\leq \mathrm{K}_{\mathrm{O}}+\mathrm{K}_{1} \mathrm{M}_{O}^{2} \mathrm{~b}^{2} \equiv \mathrm{M}_{1}
\end{aligned}
$$

where $b$ is from (2.7), and $\mathrm{M}_{0}$ is from (5.3). The constants $\mathrm{K}_{\mathrm{o}}$ and $\mathrm{k}_{1}$ are obtained by simplifying (5.15) for the given $\varepsilon$. Lemma 2.1 with $p=2$ combined with (5.16) implies 
(5.17) $\int_{\Omega}|\Phi|^{2} \mathrm{dx} \leq \mathrm{b}^{2} \int_{\Omega}\left|\Phi_{\mathrm{x}}\right|^{2} \mathrm{dx} \leq\left(4 \mathrm{~b}^{2} / \lambda\right) \mathrm{M}_{1}$.

The right hand side of (5.17) is independent of $R$, and thus, by letting $R$ approach $\infty$, we obtain

$$
\int_{\Omega}|v|^{4} d x \leq\left(4 b^{2} / \lambda\right) M_{1} .
$$

Since $\mathrm{V}^{\mathrm{i}}=0$ for $\mathrm{i} \neq \mathrm{k}, \mathrm{V}^{\mathrm{k}}=\mathrm{U}^{\mathrm{k}}$, and $\mathrm{k}$ was chosen arbitrarily, we have (5.18) true for all $U^{i}, 1 \leq i \leq N$. Summing on $i$ from 1 to $N$, we get

$$
\int_{\Omega}|\mathrm{U}|^{4} \mathrm{dx} \leq\left(4 \mathrm{~b}^{2} / \lambda\right) \mathrm{M}_{1} \mathrm{~N}^{3}
$$

where we have used the inequality

$$
|U|^{4}=\left[\sum_{i=1}^{N}\left|U^{i}\right|^{2}\right]^{2} \leq N^{2} \sum_{i=1}^{N}\left|U^{i}\right|^{4} .
$$

Now suppose $(5.8)$ holds for $s=q \geq 1$. We must show that $(5.8)$ is valid for $s=q+1$. Thus, we define $\mathrm{Z}=\Phi \psi \mathrm{V}$ with $\Phi(\mathrm{x}) \equiv \min \left\{|\mathrm{V}(\mathrm{x})|^{2(\mathrm{q}+1)}, \mathrm{R}\right\}$, $\psi(x) \equiv \min \left\{|V(x)|^{2 q}, R^{q /(q+1)}\right\}$ with $R>0$, and $V$ is defined as before. Clearly, $\mathrm{z}$ is an element of $\mathrm{H}_{0}^{1}(\Omega)$. With this value of $z$ in (1.12), we get

(5.21) $\int_{\Omega} \Phi \psi \mathrm{v}_{, \ell}^{i} C_{i j}^{\ell m} U_{, m}^{j} d x+\int_{\Omega} \Phi_{, \ell} \psi v^{i} c_{i j}^{\ell m} U_{, m}^{j} d x$

$$
\begin{aligned}
& +\int_{\Omega} \Phi \psi, \ell v^{i} C_{i j}^{\ell m} U_{, m}^{j} d x=-\int_{\Omega} \Phi \psi v_{, l}^{i} B_{i}^{\ell} d x \\
& +\int_{\Omega} \psi_{, \ell} \Phi v^{i} B_{i}^{\ell} d x+\int_{\Omega} \Phi, \ell \psi v^{i} B_{i}^{\ell} d x \\
& +\int_{\Omega} \Phi \psi v^{i} F_{i} d x .
\end{aligned}
$$


Using Assumption 5.1, we obtain

$$
\text { (5.22) } \begin{aligned}
\lambda & \int_{\Omega} \Phi \psi\left|\mathrm{v}_{\mathrm{x}}\right|^{2} \mathrm{dx}+\lambda[2(\mathrm{q}+1)]^{-1} \int_{\Omega}\left|\Phi_{\mathbf{x}}\right|^{2} \mathrm{~d} \mathbf{x} \\
& +\mathrm{q} \lambda\left[2(\mathrm{q}+1)^{2}\right]^{-1} \int_{\Omega}\left|\Phi_{\mathrm{x}}\right|^{2} \mathrm{dx} \\
& \leq \int_{\Omega} \psi \Phi\left|\mathrm{v}_{\mathrm{x}}\right|\left|\mathrm{B}_{\mathrm{k}}\right| \mathrm{d} \mathrm{x}+\int_{\Omega}\left|\psi_{\mathrm{x}}\right| \Phi|\mathrm{V}|\left|\mathrm{B}_{\mathrm{k}}\right| \mathrm{d} \mathbf{x} \\
& +\int_{\Omega} \psi\left|\Phi_{\mathrm{x}}\right||\mathrm{V}|\left|\mathrm{B}_{\mathrm{k}}\right| \mathrm{d} \mathbf{x}+\int_{\Omega} \psi \Phi|\mathrm{v}|\left|\mathrm{F}_{\mathrm{k}}\right| \mathrm{d} \mathbf{x} .
\end{aligned}
$$

We now give estimates analogous to those for the case $s=1$ for the terms on the right hand side of (5.22). We shall use the fact that $|\mathrm{V}||\Phi, \mathrm{m}|=(\Phi)^{1 / 2(q+1)}\left|\Phi_{, \mathrm{m}}\right|=$ $(\psi)^{1 / 2 q}|\Phi, m|(1 \leq m \leq n)$ throughout the estimates.

$$
\text { (5.23) } \begin{aligned}
\int_{\Omega} & |\mathrm{V}| \psi\left|\Phi_{\mathbf{x}}\right|\left|\mathrm{B}_{\mathrm{k}}\right| \mathrm{d} \mathbf{x} \leq \mathrm{M} \int_{\Omega}|\mathrm{V}| \psi\left|\Phi_{\mathbf{x}}\right| \mathrm{d} \mathbf{x} \\
& +\mathrm{M} \int_{\Omega}|\mathrm{V}| \psi\left|\Phi_{\mathbf{x}}\right||\mathrm{U}|^{1 / 2} \mathrm{~d} \mathbf{x}=\mathrm{M} \int_{\Omega} \Phi^{\delta}\left|\Phi_{\mathbf{x}}\right| \mathrm{d} \mathbf{x} \\
& +\mathrm{M} \int_{\Omega} \Phi^{\delta}\left|\Phi_{\mathrm{X}}\right||\mathrm{U}|^{1 / 2} \mathrm{dx} \\
& \leq 2 \varepsilon \int_{\Omega}\left|\Phi_{\mathbf{x}}\right|^{2} \mathrm{dx}+\left(\mathrm{M}^{2} / 4 \varepsilon\right)\left[\int_{\Omega} \Phi^{2 \delta} \mathrm{d} \mathbf{x}\right. \\
& \left.+\int_{\Omega} \Phi^{2 \delta}|\mathrm{U}| \mathrm{dx}\right] \\
\leq & 2 \varepsilon \int_{\Omega}\left|\Phi_{\mathbf{x}}\right|^{2} \mathrm{dx}+2 \varepsilon \int_{\Omega}|\Phi|^{2} \mathrm{dx}
\end{aligned}
$$




$$
\begin{gathered}
+[1 /(2 q+2)][\delta / \varepsilon]^{2 q+1}\left[M^{2} / 4 \varepsilon\right]^{2 q+2}[\text { mes } \Omega \\
\left.+\int_{\Omega}|U|^{2 q+2} d x\right] \\
\leq \quad 2 \varepsilon\left(1+b^{2}\right) \int_{\Omega}\left|\Phi_{x}\right|^{2} d x \\
+[1 /(2 q+2)][\delta / \varepsilon]^{2 q+1}\left[M^{2} / 4 \varepsilon\right]^{2 q+2}[\text { mes } \Omega \\
\left.+\int_{\Omega}|U|^{2 q+2} d x\right]
\end{gathered}
$$

with $\delta \equiv(2 q+1) /(2 q+2)$. Since $q \geq 1$, it is clear that $2 q+2$ $\leq 4 \mathrm{q}$ which implies that the last integral in (5.23) converges by the induction hypothesis. Continuing with the estimates, we have

(5.24) (2q+1) $\int_{\Omega} \Phi \psi\left|\mathrm{V}_{\mathbf{x}}\right|\left|\mathrm{B}_{\mathrm{k}}\right| \mathrm{dx} \leq(2 \mathrm{q}+1) \mathrm{M}\left[\int_{\Omega} \Phi \psi\left|\mathrm{V}_{\mathbf{x}}\right| \mathrm{dx}\right.$

$$
\begin{aligned}
& \left.+\int_{\Omega} \Phi \psi\left|\mathrm{v}_{\mathrm{x}}\right||\mathrm{U}|^{1 / 2} \mathrm{dx}\right] \\
& \leq 2 \varepsilon \int_{\Omega} \Phi \psi\left|\mathrm{v}_{\mathrm{x}}\right|^{2} \mathrm{dx}+(2 \mathrm{q}+1)^{2} \mathrm{M}^{2} / 4 \varepsilon\left[\int_{\Omega} \Phi \psi \mathrm{dx}\right. \\
& \left.+\int_{\Omega} \Phi \psi|\mathrm{U}| \mathrm{dx}\right] \\
& \leq 2 \varepsilon \int_{\Omega} \Phi \psi\left|\mathrm{v}_{\mathrm{x}}\right|^{2} \mathrm{dx}+2 \varepsilon \int_{\Omega}|\Phi|^{2} \mathrm{dx} \\
& +[1 /(2 \mathrm{q}+2)][\delta / \varepsilon]^{2 \mathrm{q}+1}\left[(2 \mathrm{q}+1)^{2} \mathrm{M}^{2} / 4 \varepsilon\right]^{2 \mathrm{q}+2} \text { [mes } \Omega \\
& \left.+\int_{\Omega}|\mathrm{U}|^{2 \mathrm{q}+2} \mathrm{dx}\right]
\end{aligned}
$$


$\leq 2 \varepsilon \int_{\Omega} \Phi \psi\left|\mathrm{v}_{\mathbf{x}}\right|^{2} \mathrm{dx}+2 \varepsilon \mathrm{b}^{2} \int_{\Omega}\left|\Phi_{\mathbf{x}}\right|^{2} \mathrm{dx}$

$+[1 /(2 q+2)][\delta / \varepsilon]^{2 q+1}\left[(2 q+2)^{2} M^{2} / 4 \varepsilon\right]^{2 q+2}[$ mes $\Omega$

$$
\left.+\int_{\Omega}|u|^{2 q+2} d x\right]
$$

Finally, the last term in (5.22) admits the estimate

(5.25) $\int_{\Omega} \psi \Phi|\mathrm{V}|\left|\mathrm{F}_{\mathrm{k}}\right| \mathrm{dx} \leq \mathrm{M}\left[\int_{\Omega} \psi \Phi|\mathrm{V}| \mathrm{dx}\right.$

$$
\begin{aligned}
&\left.+\int_{\Omega} \Phi \psi|\mathrm{V}||\mathrm{U}|^{1 / 2} \mathrm{dx}\right] \\
&= \mathrm{M}\left[\int_{\Omega} \Phi^{2 \delta}|\mathrm{V}| \mathrm{dx}+\int_{\Omega} \Phi^{2 \delta}|\mathrm{V}||\mathrm{U}|^{1 / 2} \mathrm{dx}\right] \\
& \leq \varepsilon \int_{\Omega}|\Phi|^{2} \mathrm{dx}+\varepsilon \int_{\Omega} \Phi^{2 \delta}|\mathrm{V}|^{2} \mathrm{dx} \\
&+[1 /(2 \mathrm{q}+2)][\delta / \varepsilon]^{2 q+1} \mathrm{M}^{2 \mathrm{q}+2} \int_{\Omega}|\mathrm{V}|^{2 \mathrm{q}+2} \mathrm{dx} \\
&+\left(\mathrm{M}^{2} / 4 \varepsilon\right) \int_{\Omega} \Phi^{2 \delta}|\mathrm{U}| \mathrm{dx} \\
& \leq[1 /(2 \mathrm{q}+2)][\delta / \varepsilon]^{2 q+1}\left\{\mathrm{M}^{2 q+2} \int_{\Omega}|\mathrm{V}|^{2 \mathrm{q}+2} \mathrm{dx}\right. \\
&\left.+\left(\mathrm{M}^{2} / 4 \varepsilon\right)^{2 q+2} \int_{\Omega}|\mathrm{U}|^{2 \mathrm{q}+2} \mathrm{dx}\right\}+\varepsilon \int_{\Omega}|\Phi|^{2} \mathrm{dx} \\
&+\varepsilon \int_{\Omega} \Phi^{2 \delta}|\mathrm{V}|^{2} \mathrm{dx} .
\end{aligned}
$$

To estimate the last term in $(5.25)$, we use the inequality 
(5.26) $\left|\left[\left(\Phi^{2 \delta}|\mathrm{v}|^{2}\right)^{1 / 2}\right], \mathrm{m}\right|^{2} \leq 2(2 \mathrm{q}+1)^{2}[\Phi(2 \mathrm{q}-1) /(\mathrm{q}+1)$

$$
\begin{aligned}
& \left.+\Phi^{2 \delta}\right]\left|v_{x}\right|^{2} \leq 2(2 q+1)^{2}\left[\Phi^{2 \delta}+A(q)+\Phi \psi\right]\left|v_{x}\right|^{2} \\
& =2(2 q+1)^{2}[2 \psi \Phi+A(q)]\left|v_{x}\right|^{2}
\end{aligned}
$$

where $A(q)=[2 /(2 q+1)][(2 q-1) /(2 q+1)]^{(2 q-1) / 2}$. Combining (5.26) and (5.25), we get

(5.27) $\int_{\Omega} \psi \Phi|\mathrm{V}| \quad\left|\mathrm{F}_{\mathrm{k}}\right| \mathrm{dx} \leq 2 \varepsilon \mathrm{b}^{2} \int_{\Omega}\left|\Phi_{\mathbf{x}}\right|^{2} \mathrm{dx}$

$$
\begin{aligned}
& +[1 /(2 q+2)][\delta / \varepsilon]^{2 q+1}\left\{M^{2 q+2} \int_{\Omega}|v|^{2 q+2} d x\right. \\
& \left.+\left(M^{2} / 4 \varepsilon\right)^{2 q+2} \int_{\Omega}|U|^{2 q+2} d x\right\} \\
& +2 b^{2}(2 q+1)^{2} A(q) \int_{\Omega}\left|v_{x}\right|^{2} d x .
\end{aligned}
$$

We may now combine (5.22) through (5.24) with (5.27) and choose $\varepsilon=\varepsilon^{\prime}>0$ sufficiently small and simplify to get

$$
\text { (5.28) } \begin{aligned}
\int_{\Omega} \Phi \psi\left|\mathrm{v}_{\mathrm{x}}\right|^{2} \mathrm{dx}+\int_{\Omega}|\Phi|^{2} \mathrm{dx} \leq \mathrm{K}_{2}\left(\mathrm{q}, \varepsilon^{\prime}, \mathrm{M}, \mathrm{n}, \mathrm{b}, \Omega\right) \\
+\mathrm{K}_{3}\left(\mathrm{q}, \varepsilon^{\prime}, \Omega, \mathrm{n}, \mathrm{b}\right)\left[\int_{\Omega}|\mathrm{U}|^{2 \mathrm{q}+2} \mathrm{dx}+\int_{\Omega}\left|\mathrm{v}_{\mathrm{x}}\right|^{2}+1\right] .
\end{aligned}
$$

However, by the induction hypothesis, the integrals on the right hand side of $(5.28)$ are bounded by a constant $\tilde{M}(q)$ depending on $\mathrm{q}$ and known constants. Thus, we have a constant $\bar{M}(q)$ depending only on known constants and $q$ such 
that

(5.29) $\quad \int_{\Omega}\left|\Phi_{x}\right|^{2} d x \leq \bar{M}(q)$.

Lemma 2.1 now implies

$$
\int_{\Omega}|\Phi|^{2} d x \leq b^{2} \bar{M}(q) \equiv \overline{\bar{M}}(q+1) .
$$

We now let $R$ approach $\infty$ in $(5.30)$ and obtain

$$
\int_{\Omega}|\mathrm{V}|^{4(q+1)} \mathrm{dx} \leq \overline{\overline{\mathrm{M}}}(\mathrm{q}+1) .
$$

Since $\mathrm{k}$ was chosen arbitrarily between 1 and $\mathrm{N}$, we have that (5.31) is valid for $U^{i}=V, 1 \leq i \leq N$. By summing on $i$ from 1 to $N$ in (5.31), we obtain (5.8) for $s=q+1$ whenever it is true for $s=q$. Thus, by induction, we have that (5.8) is true for all $s \geq 1$, and the proof is complete.

THEOREM 5.1. Suppose Assumption 5.1 is valid. Then there exists a constant $\mathrm{K}$ depending only on known constants such that

$$
\underset{x \varepsilon \Omega}{\operatorname{ess} \sup }|U(x)| \leq K
$$

PROOF. Let $r$ be an arbitrary integer between 1 and $\mathrm{N}$ and put $\mathrm{V}=\mathrm{U}^{\mathrm{r}}$. In (1.12) let $\mathrm{z}^{\mathrm{i}}(\mathrm{x})=0$ if $\mathrm{i} \neq \mathrm{r}$ and $\mathrm{Z}^{\mathrm{r}}(\mathrm{x})=\max \{\mathrm{V}(\mathrm{x})-\mathrm{k}, 0\}$ where $\mathrm{k} \geq 1$. From [8, p. 53], we have $\mathrm{z}$ in $\mathrm{H}_{0}^{l}(\Omega)$. Substituting $\mathrm{z}$ into (1.12) yields

$$
\int_{A} z_{, l}^{i} C_{i j}^{\ell m} U_{, m}^{j} d x+\int_{A} z_{, l}^{i} B_{i}^{l} d x=\int_{A} z^{i} F_{i} d x
$$

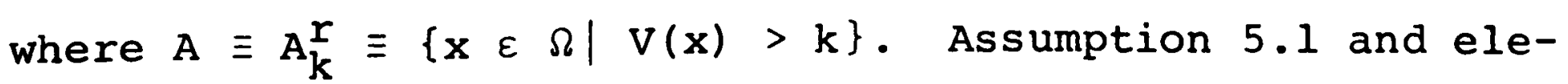


mentary estimates using Lemma 2.2 yield

(5.34) $\lambda \int_{A}\left|V_{x}\right|^{2} d x \leq \int_{A}\left|V_{x}\right||B| d x+\int_{A}(V-k)|F| d x$$$
\leq \varepsilon \int_{\mathrm{A}}\left|\mathrm{V}_{\mathrm{x}}\right|^{2} \mathrm{dx}+(4 \varepsilon)^{-1} \int_{\mathrm{A}}|\mathrm{B}|^{2} \mathrm{dx}
$$$$
+\int_{A}|F|^{2} d x+\int_{A}(V-k)^{2} d x .
$$

We use Assumption 5.1 to get the following estimates:

$$
\begin{gathered}
(4 \varepsilon)^{-1} \int_{\mathrm{A}}|\mathrm{B}|^{2} \mathrm{dx} \leq\left(\mathrm{M}^{2} / 2 \varepsilon\right) \int_{\mathrm{A}}\left[1+|\mathrm{U}|^{2 \alpha}\right] \mathrm{dx} \\
=\left(\mathrm{M}^{2} / 2 \varepsilon\right)\left[\int_{\mathrm{A}}|\mathrm{U}|^{2 \alpha} \mathrm{dx}+\operatorname{mes} \mathrm{A}\right] .
\end{gathered}
$$

(5.36) $\int_{A}|F|^{2} d x \leq 2 M^{2} \int_{A}\left[1+U^{2 \alpha}\right] d x$

$$
=2 \mathrm{M}^{2}\left[\int_{\mathrm{A}}|\mathrm{U}|^{2 \alpha} \mathrm{dx}+\operatorname{mes} \mathrm{A}\right] .
$$

In (5.34) put $\varepsilon=\lambda / 2$ and use (5.35) and (5.36) to get

$$
\text { (5.37) } \begin{aligned}
\int_{A}\left|v_{x}\right|^{2} d x & \leq(2 / \lambda) \int_{A}(V-k)^{2} d x \\
& +\left[4 M^{2}(\lambda+1) / \lambda^{2}\right]\left[\text { mes } A+\int_{A}|U|^{2 \alpha} d x\right]
\end{aligned}
$$

where $M$ is from (5.1). We now use Lemma 5.1 and Lemma 5.2 to estimate the last integral in (5.37). First, we consider the cases $\mathrm{n}=3,4$, or 5 . Lemma 2.1 and Lemma 5.1 imply the existence of a constant $M_{1}$ depending only upon known constants such that 
$(5.38)$

$$
\int_{\Omega}|\mathrm{V}|^{\mathrm{P}} \mathrm{dx} \leq \int_{\Omega}|\mathrm{U}|^{\mathrm{P}} \mathrm{dx} \leq \mathrm{M}_{1}
$$

for $1 \leq p \leq 2 n /(n-2)$. Thus, with the use of Hölder's inequality, we obtain

(5.39) $\int_{A}|v|^{2 \alpha} d x \leq\left[\int_{A}|V|^{2 \alpha q} d x\right]^{1 / q}[\text { mes } A]^{1-(1 / q)}$

where $q=n /[\alpha(n-2)]$ for $n=3,4$, or 5 . Using (5.38) in (5.39), we have

(5.40) $\quad \int_{A}|V|^{2 \alpha} d x \leq M_{1}^{\alpha(n-2) / n}[\text { mes } A]^{1-[\alpha(n-2) / n]}$

From Assumption 5.1 , we get $-\alpha(n-2) / n>-2 / n$ which implies the existence of $\varepsilon_{0}>0$ such that $1-[\alpha(n-2) / n]=1-(2 / n)+\varepsilon_{0}$. Combining this with $(5.39)$ and $(5.40)$, we have

$$
\text { (5.41) } \begin{aligned}
\int_{A}\left|v_{x}\right|^{2} d x & \leq(2 / \lambda) \int_{A}(V-k)^{2} d x \\
& +\left[4 M^{2}(\lambda+1) / \lambda^{2}\right]\{\text { mes } A \\
& \left.+M_{1}^{[\alpha(n-2) / n]}[\text { mes } A] 1-(2 / n)+\varepsilon_{0}\right\} \\
\leq\left\{\left[4 M^{2}(\lambda+1) / \lambda^{2}\right]+M_{1}^{\alpha}(n-2) / n\right. & +2 / \lambda\}\left\{\int_{A}(V-k)^{2} d x\right. \\
& +k^{2}(\operatorname{mes} A)+k^{2}(\operatorname{mes} A)
\end{aligned}
$$

From the definition of $\mathrm{V}$ and $\mathrm{A}$, we may conclude from Theorem 2.5 that there exists a constant $k_{0}$ depending only on known constants such that

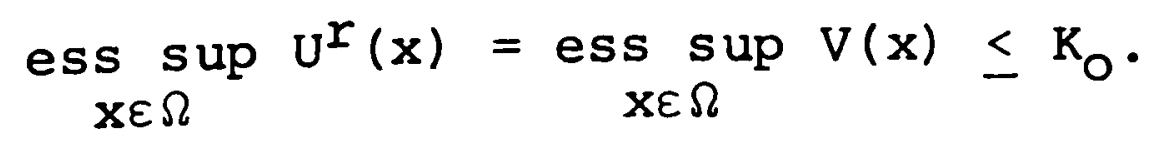


To obtain a bound for ess $\sup _{\mathrm{x}}\left(-\mathrm{U}^{\mathrm{r}}(\mathrm{x})\right)$, we multiply (1.12) by -1 to get

(5.43) $\int_{\Omega} z_{, l}^{i} C_{i j}^{l m}\left(-U^{j}\right), m d x-\int_{\Omega} z_{, l}^{i} B_{i}^{l} d x+\int_{\Omega} z^{i} F_{i} d x=0$.

We now put $z^{i}=0$ for $i \neq r$ and $z^{r}(x)=\max \{-V(x)-k, 0\}$ with $\mathrm{k} \geq 1$. By repeating the analysis above with $\mathrm{V}$ replaced with $-v$, we obtain a constant $k_{1}$ depending only on known constants such that

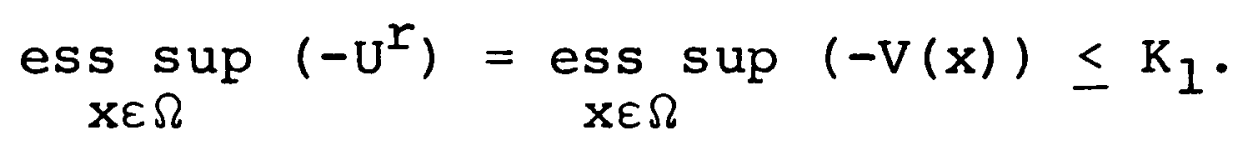

Since $r$ was chosen arbitrarily between $I$ and $N$, we have (5.44) true for all such $r$. Therefore, (5.32) is proven for $\mathrm{n}=3,4$, or 5 . If $\mathrm{n}=2$, choose $\mathrm{q}=1 / \alpha$ in $(5.39)$, and $(5.41)$ becomes

$$
\text { (5.45) } \begin{aligned}
\int_{A}\left|V_{x}\right|^{2} d x \leq & \left\{\left[4 M^{2}(\lambda+1) / \lambda^{2}\right]+M_{1}^{\alpha}+2 / \lambda\right\} \\
\times & {\left[\int_{A}(V-k)^{2} d x+k^{2}(\text { mes } A)+\right.} \\
& k^{2}\left(\text { mes A) }{ }^{1-\alpha}\right],
\end{aligned}
$$

and the proof continues as in the first case. For $\mathrm{n} \geq 6$, choose $q=4 \mathrm{~s}$ where $\mathrm{s}$ is the first integer greater than $\mathrm{n} / 8$. Thus, $\mathrm{q}=4 \mathrm{~s}>\mathrm{n} / 2$, and there exists an $\varepsilon_{0}>0$ such that $1-(1 / q)=1-(2 / n)+\varepsilon_{0}$, and $(5.40)$ now becomes

(5.46) $\int_{A}|v|^{2 \alpha} d x=\int_{A}|V| d x \leq[\overline{\bar{M}}(s)]^{1 / 4 s}(\operatorname{mes} A)^{1-(2 / n)+\varepsilon_{O}}$ where $\overline{\bar{M}}(s)$ is from (5.8). With this inequality, (5.41) now 
has the form

$$
\text { (5.47) } \begin{aligned}
\int_{A}\left|V_{x}\right|^{2} d x \leq & \left\{\left[4 M^{2}(\lambda+1) / \lambda^{2}\right]+[\overline{\bar{M}}(s)] 1 / 4 s+2 / \lambda\right\} \\
& \times\left\{\int_{A}(V-k)^{2} d x+k^{2}(\operatorname{mes} A)\right. \\
& +k^{2}(\operatorname{mes} A)^{\left.1-(2 / n)+\varepsilon_{O}\right\}}
\end{aligned}
$$

and the analysis continues as in the two previous cases. This completes the proof.

The condition $\alpha<1$ in Assumption 5.1 cannot in general be extended to $\alpha=1$. Consider the following examples:

EXAMPLE 1. Suppose $N=1, B_{1}^{l}[\mathrm{x}, \mathrm{U}]=0$ for all $\ell=1$, $2, \ldots, n, F_{I}[x, U]=n U, C_{1 l}^{l m}[x, U]=\delta_{m}^{l} \equiv$ Kronecker delta, and $\Omega=(0, \pi) \times(0, \pi) \times \cdots \times(0, \pi)$ with the product taken over $n$ intervals. Then, for any constant $K$, $U(x)=K\left(\sin \left(x_{1}\right)\right)\left(\sin \left(x_{2}\right)\right) \cdots\left(\sin \left(x_{n}\right)\right)$ is a solution of $(1.1)$ and $(1.2)$

EXAMPLE 2. Suppose $\mathrm{n}=1, \mathrm{~N}=2, \mathrm{~B}_{1}^{1}[\mathrm{x}, \mathrm{U}]=-\mathrm{U}_{2}, \mathrm{~B} \frac{1}{2}[\mathrm{x}, \mathrm{U}]$ $=U_{1}, F_{i}[x, U]=0, C_{i j}^{11}[x, U]=\delta_{j}^{i}$ for $i, j=1,2$, and $\Omega$ is $(0,2 \pi)$. Then, for any constant $K, U(x)=K\left(\begin{array}{l}\sin (x) \\ \cos (x)-1\end{array}\right)$ is a solution of (1.1) and (1.2). 


\section{CHAPTER VI}

BOUNDEDNESS OF THE HÖLDER NORM

We can now find a bound for the Hölder norm of weak solutions of (1.1) and (1.2) under the restrictions of Assumption 5.1. The proof follows closely that of Ladyzhenskaya and Ural'tseva [8, pp. 249-251] for a single equation .

THEOREM 6.1. Suppose Assumption 5.1 is valid. Then an arbitrary weak solution $U$ of (1.1) and (1.2) belongs to $C_{0, \beta}(\bar{\Omega})$ with exponent $\beta>0$ depending on $M_{0} \equiv$ ess $\sup _{x \in \Omega}|U(x)|$ and known constants. The quantity $|U|_{\beta, \Omega}$ is bounded above by an expression determined by $\lambda$ and $M_{C}$ from (3.2) and the constants $a_{0}$ and $\theta_{0}$ in the definition of condition (A).

PROOF. We shall show that $\mathrm{U}^{\mathrm{r}}$ belongs to some class $B\left(\bar{\Omega}, M_{O}, \gamma, \delta, 0\right)$ for arbitrary $r$ between 1 and $N$. In (1.12) let $z^{i}(x)=0$ for $i \neq r$ and $z^{r}(x)=[\zeta(x)]^{2} \max \{V(x)-k, 0\}$ where $\mathrm{V}=\mathrm{U}^{\mathrm{r}}, \mathrm{k} \geq 0$, and $\zeta$ is a smooth function of compact support with range in $[0,1]$ on some sphere $\mathrm{K}_{\rho}$.

Observe that $\mathrm{z}^{\mathrm{r}}$ is nonzero only on the set $\mathrm{A} \equiv \mathrm{A}_{\mathrm{k}, \rho}^{\mathrm{r}} \equiv$ $\left\{x \in K_{\rho} \cap \Omega \mid V(x)>k\right\}$, and thus, (1.12) becomes

$$
\begin{aligned}
& \text { (6.1) } \int_{A} \zeta^{2} c_{r r}^{\ell m} v_{, l} v_{, m} d x+\int_{A} 2 \zeta \zeta, l c_{r r}^{\ell m} v, m(V-k) d x \\
& =-\int_{A} \zeta^{2} V_{, l} B_{r}^{l} d x-\int_{A} 2 \zeta \zeta, l(V-k) B_{r}^{l} d x \\
& +\int_{A} \zeta^{2}(V-k) F_{r} d x
\end{aligned}
$$


Using Assumption 5.1 and the boundedness of $|U|$ from Theorem 5.1, we get

$$
\text { (6.2) } \begin{aligned}
& \lambda \int_{A} \zeta^{2}\left|v_{x}\right|^{2} d x \leq 2 M \int_{A} \zeta\left|\zeta_{x}\right|(V-k)\left|v_{x}\right| d x \\
&+M\left[1+M_{O}^{\alpha}\right] \int_{A} \zeta^{2}\left|v_{x}\right| d x \\
&+2 M\left[1+M_{O}^{\alpha}\right] \int_{A} \zeta\left|\zeta_{x}\right|(V-k) d x \\
&+M\left[1+M_{O}^{\alpha}\right] \int_{A} \zeta^{2}(V-k) d x .
\end{aligned}
$$

With the use of (2.8), we get the following estimates for the above integrals.

(6.3) $2 M_{C} \int_{A} \zeta\left|\zeta_{x}\right|(V-k)\left|v_{x}\right| d x$

$$
\leq \varepsilon \int_{A} \zeta^{2}\left|V_{x}\right|^{2} d x+\left(M_{C}^{2} / \varepsilon\right) \int_{A}\left|\zeta_{x}\right|^{2}(V-k)^{2} d x
$$

(6.4) $M\left[1+M_{O}^{\alpha}\right] \int_{A} \zeta^{2}\left|v_{x}\right| d x$

$$
\leq \varepsilon \int_{A} \zeta^{2}\left|\mathrm{~V}_{\mathrm{x}}\right|^{2} \mathrm{dx}+\left\{\mathrm{M}^{2}\left[1+\mathrm{M}_{\mathrm{O}}^{\alpha}\right] 2 / 4 \varepsilon\right\} \int_{\mathrm{A}} \zeta^{2} \mathrm{dx} .
$$

(6.5) $2 M\left[1+M_{0}^{\alpha}\right] \int_{A} \zeta\left|\zeta_{X}\right|(V-k) d x$

$$
\leq(1 / 2) \int_{A}\left|\zeta_{x}\right|^{2}(V-k)^{2} d x+2 M^{2}\left[1+M_{O}^{\alpha}\right]^{2} \int_{A} \zeta^{2} d x .
$$

Using (6.2) through (6.5), we get 
(6.6) $\lambda \int_{A} \zeta^{2}\left|v_{x}\right|^{2} d x \leq 2 \varepsilon \int_{A} \zeta^{2}\left|v_{x}\right|^{2} d x$

$$
\begin{aligned}
& +\left[\left(M_{C}^{2} / \varepsilon\right)+(1 / 2)\right] \int_{A}\left|\zeta_{X}\right|^{2}(V-k)^{2} d x \\
& +\left[1+M_{O}^{\alpha}\right]{ }^{2} M^{2}[(1 / 4 \varepsilon)+2] \int_{A} \zeta^{2} d x \\
& +M\left[1+M_{O}^{\alpha}\right] \int_{A} \zeta^{2}(V-k) d x
\end{aligned}
$$

For values of $\mathrm{k}$ such that $\sup _{\mathrm{x} \in \mathrm{K} \cap \cap \Omega} \mathrm{V} \cap(\mathrm{x})-\mathrm{k} \leq \delta \equiv 1$, and $\varepsilon=\lambda / 4$, we get

$$
\text { (6.7) } \begin{gathered}
\int_{A} \zeta^{2}\left|v_{x}\right|^{2} d x \leq K\left(\alpha, M_{O}, M, \lambda, M_{C}\right)\left[\int_{A}\left|\zeta_{X}\right|^{2}(V-k)^{2} d x\right. \\
\left.+\int_{A} \zeta^{2} d x\right]
\end{gathered}
$$

where $\mathrm{K}\left(\alpha, \mathrm{M}_{\mathrm{O}}, \mathrm{M}, \lambda, \mathrm{M}_{\mathrm{C}}\right) \equiv\left(2 / \lambda^{2}\right)\left[4 \mathrm{M}_{\mathrm{C}}^{2}+(\lambda / 2)+\lambda\left[1+\mathrm{M}_{\mathrm{O}}^{\alpha}\right]^{2} \mathrm{M}_{\mathrm{O}}^{2}[1+2 \lambda]\right.$ $\left.+\lambda M\left[1+M_{0}^{\alpha}\right]\right\}$. Now, if we take the function $\zeta$ equal to unity in a sphere $k_{\rho-\sigma \rho}$ where $\sigma \varepsilon(0,1)$, that is concentric with the sphere $K_{\rho}$ in such a way that (See [8, p. 250].)

$$
\left|\zeta_{\mathbf{x}}\right| \leq \mathrm{E} / \sigma \rho
$$

for some constant $E$, then (6.7) yields the series of inequalities

$$
\text { (6.9) } \begin{aligned}
\int_{A_{k}}^{r}\left|V_{x}\right|^{2} d x \leq K\left(\alpha, M_{O}, M, \lambda, M_{\Xi}\right) & \left\{\left[1 /(\sigma \rho)^{2}\right] \underset{x \in A_{k, \rho}^{r}}{r}\{V(x)-k\}^{2}\right. \\
& +1\} \text { mes } A_{k, l}^{r}
\end{aligned}
$$

that determine the class $B\left(\bar{\Omega}, M_{O}, \gamma, \delta, 0\right)$. 
We obtain analogous inequalities for the function $-V$ if we put $z^{r}(x)=[\zeta(x)]^{2} \max \{-V(x)-k, 0\}$ where the values of $\mathrm{k}$ are such that $\sup _{x \in \mathrm{K} \cap \Omega}(-\mathrm{V}(\mathrm{x}))-\mathrm{k} \leq \delta \equiv 1$ and $\mathrm{k} \geq 0$. Since $r$ was chosen arbitrarily between 1 and $N$, we have $\mathrm{U}^{i}$ in $\mathrm{B}\left(\bar{\Omega}, \mathrm{M}_{O}, \gamma, \delta, 0\right)$ for each $i=1,2, \ldots, N$. Therefore, Theorem 2.4 implies Theorem 6.1, and the proof is complete. 
CHAPTER VII

CONCLUSION

The principal result of Chapter III is Theorem 3.4. The restrictions on $C, B$, and $F$ needed to achieve this existence theorem seem quite weak with the possible exception of (3.38) limiting the size of $\varepsilon_{1}$. However, to allow $\varepsilon_{1}$ to be arbitrary, it is likely that the method used here would have to be altered significantly.

The results of Chapter IV seem adequate since, in the case of $n=N=1$, it reduces to the restrictions involved in the two-point boundary value problem studied by Ford [5] .

In Chapter V and Chapter VI, we have used the methods that Ladyzhenskaya and Ural'tseva [8] developed for a single quasilinear equation and for linear systems to obtain some regularity results for our weak solutions. Although the necessity of having $\alpha<1$ in Assumption 5.1 is demonstrated by an example, the condition that $\alpha$ decrease as $n$ increases up to $n=5$ and then equal $1 / 2$ for $\mathrm{n} \geq 6$ seems unnatural. It would also be desirable to prove analogous results for a more general form for $c_{i j}^{\ell m}$. 


\section{LIST OF REFERENCES}

[1] L. Bers, F. John, and M. Schechter, Partial Differential Equations, Wiley (Interscience), New York, 1964.

[2] F. E. Browder, Functional analysis and partial differential equations, I, Annals of Math. 138 (1959), 5579 .

[3] F. E. Browder, Existence theory of boundary value problems for quasilinear elliptic systems with strongly nonlinear lower order terms, Partial Differential Equations (Proc. Sympos. Pure Math., Vol. XXIII, Univ. of Calif., Berkeley, Calif., 1971), 269-286, Amer. Math. Soc., Providence, 1973.

[4] J. R. Cannon, W. T. Ford, and A. V. Lair, Quasilinear parabolic systems, J. of Differential Equations ( to appear).

[5] W. T. Ford, on the first boundary value problem for $\left[h\left(x, x^{\prime}, t\right)\right]^{\prime}=f\left(x, x^{\prime}, t\right)$, Proc. of the Amer. Math. Soc. 35 (1972), 491-497.

[6] G. H. Hardy, J. E. Littlewood, and G. Polya, Inequalities, 2nd ed., Cambridge Univ. Press, New York, 1952.

[7] A. V. Ivanov, The solvability of the Dirichlet problem for certain classes of second order elliptic systems, Proc. of the Steklov Inst. Math. 125 (1973), 49-79.

[8] O. A. Ladyzhenskaya and N. N. Ural'tseva, Linear and Quasilinear Elliptic Equations, Academic Press, New York, 1968 .

[9] A. V. Lair, A Rellich compactness theorem for sets of finite volume, Amer. Math. Monthly (to appear).

[10] C. Miranda, Partial Differential Equations of Elliptic Type, 2nd rev. ed., Springer-Verlag, New York, 1970.

[11] H. L. Royden, Real Analysis, 2nd ed., Macmillan, New York, 1968 .

[12] J. T. Schwartz, Nonlinear Functional Analysis, Gordon and Breach, New York, 1969. 
[13] M. I. Visik, Quasi-linear strongly elliptic systems of differential equations in divergence form, Trans. Mos cow Math. Soc. 1963, 148-208.

[14] K. Yosida, Functional Analysis, 2nd ed., SpringerVerlag, New York, 1968. 